\title{
Exactly-solvable models derived from a generalized Gaudin algebra
}

\author{
G. Ortiz ${ }^{a}$, R. Somma ${ }^{\text {a }}$ J. Dukelsky ${ }^{\text {b }}$, S. Rombouts ${ }^{c}$ \\ ${ }^{a}$ Los Alamos National Laboratory, Los Alamos, New Mexico 87545, USA \\ b Instituto de Estructura de la Materia, CSIC, Serrano 123, 28006 Madrid, Spain \\ c Ghent University, Department of Subatomic and Radiation Physics, Proeftuinstraat 86, B-9000 Gent, Belgium \\ Received 19 July 2004; received in revised form 20 October 2004; accepted 9 November 2004
}

Available online 23 November 2004

\begin{abstract}
We introduce a generalized Gaudin Lie algebra and a complete set of mutually commuting quantum invariants allowing the derivation of several families of exactly solvable Hamiltonians. Different Hamiltonians correspond to different representations of the generators of the algebra. The derived exactly-solvable generalized Gaudin models include the Hamiltonians of Bardeen-Cooper-Schrieffer, Suhl-Matthias-Walker, Lipkin-Meshkov-Glick, the generalized Dicke and atom-molecule, the nuclear interacting boson model, a new exactly-solvable Kondo-like impurity model, and many more that have not been exploited in the physics literature yet.

(C) 2004 Elsevier B.V. All rights reserved.
\end{abstract}

PACS: 02.30.Ik; 03.65.Fd; 21.60.Fw; 74.20.Fg; 75.10.Jm

\section{Introduction}

During last decade we have witnessed an enormous progress both in low-temperature experimental techniques and in the design and better characterization of novel materials and cold atomic systems. These developments allow one to explore the quantum world in a more fundamental way. In particular, since interactions between particle constituents

E-mail addresses: ortiz@viking.lanl.gov (G. Ortiz), somma@viking.lanl.gov (R. Somma), dukelsky@iem.cfmac.csic.es (J. Dukelsky), stefan.rombouts@rug.ac.be (S. Rombouts). 
can lead to unexpected phenomena, one would like to achieve sufficient degree of quantum control to take advantage of it. Theoretical work on strongly coupled systems is helping in this regard. For example, research on critical phenomena in quantum phase transitions, where the Landau-Ginzburg paradigm of broken-symmetry phase transitions does not apply, shows interesting scenarios. Such is the case of the recently proposed deconfined quantum critical points where fractionalized excitations may emerge at criticality with observable consequences [1]. A crucial theoretical bottleneck, however, is the lack of exactly-solvable interacting many-body models, since nonperturbative and nonlinear phenomena play a relevant role.

The main goal of this paper is to introduce a generalization of the Gaudin algebra [2], which we name generalized Gaudin algebra (GGA), whose quantum invariants can be exactly diagonalized and may be related to Hamiltonian operators of exactly-solvable problems of interacting constituents. By exactly-solvable model we mean a model Hamiltonian whose entire spectral problem is reduced to an algebraic one (i.e., it is explicitly diagonalized), a fact that is associated to the existence of a certain hidden symmetry in the model under consideration. There are larger classes of Hamiltonians characterized by exact solvability of only certain part of their spectra; these are called quasiexactly solvable [3], and the $t-J_{z}$ chain model is an example [4]. Clearly, exactly-solvable models may be used as a starting point to construct many other quasiexactly solvable models.

As we will see, we have identified the main operator algebra underlying the integrability and exact solvability of many well-known models, thus unifying their description in a single algebraic framework. Simply diagonalizing the quantum invariants of the GGA is sufficient to solve all those problems, which include the Bardeen-Cooper-Schrieffer (BCS) [5], Suhl-Matthias-Walker (SMW) [6], Lipkin-Meshkov-Glick (LMG) [7], generalized Dicke (GD) [8], and many others of interest in condensed-matter, molecular, atomic and nuclear physics. The basic point is that all these various models, which form the general class of $X Y Z$ Gaudin models, can be derived using different realizations of the generators of the GGA. For example, the BCS model is obtained from the quantum invariants of the GGA after representing their generators in terms of fermionic-pair realizations of the generators of $\bigoplus_{\mathrm{I}} s u(2)$. A consequence of this unification is that new exactly-solvable models can be realized after a proper representation of the GGA. For instance, one can write down exactly-solvable $S U(N)$ spin and mixed representation models, such as spin-fermion, spinboson or fermion-boson Hamiltonians.

We start by defining the GGA in Section 2. We show how the $X Y Z$ Gaudin equation naturally emerges from the Jacobi identity for the generators of the GGA. We also introduce the quantum invariants that will serve as the generating functions for all conserved quantities of the generalized (integrable) $X Y Z$ Gaudin models.

In Section 3 the $X X Z$ Gaudin equation and the diagonalization of the $X X Z$ Gaudin models are studied. We show a family of solutions of the $X X Z$ Gaudin equation, which includes the well-known rational, trigonometric, hyperbolic $[9,10]$ and the new solution found by Richardson [11] as especial limits. In particular, we show that the latter can be considered as a reparametrization of the other three. The main use of these solutions is to design exactly-solvable Hamiltonians with a large set of free parameters, thus providing additional freedom to tune interactions. 
Table 1

Exactly-solvable models (most of them discussed in this paper) which are derived from different representations of the generators of the generalized Gaudin algebra (GGA). 1 refers to the number of copies of the algebra used to write down the model. Notice that some models correspond to mixed algebraic representations of the generators of the GGA. Notation: F: fermionic, B: bosonic, S: spin, P: pseudospin, $h_{4}$ : Heisenberg-Weyl algebra

\begin{tabular}{|c|c|c|c|}
\hline Gaudin algebra & Representation & 1 & Model \\
\hline \multirow[t]{7}{*}{$X X X$} & $\bigoplus_{\mathbf{l}} s u(2)-\mathrm{F}-\mathrm{P}$ & & BCS Richardson \\
\hline & & $N$ & Nuclear pairing \\
\hline & & & $\mathrm{BCS}(\mathbf{k} \uparrow,-\mathbf{k} \downarrow)$ \\
\hline & $\bigoplus_{\mathbf{l}} s u(2)-\mathrm{F}-\mathrm{S}$ & $N$ & Particle-hole-like \\
\hline & $\bigoplus_{\mathbf{l}} s u(1,1)-\mathrm{B}$ & $N$ & B BCS \\
\hline & $\bigoplus_{\mathbf{l}} s u(2) \oplus s u(2)$ & $N$ & Central spin \\
\hline & $\bigoplus_{\mathbf{l}} s u(1,1) \oplus s u(1,1)$ & $N$ & B central spin \\
\hline \multirow[t]{8}{*}{$X X Z$} & $\bigoplus_{\mathbf{l}} s u(2)-\mathrm{F}-\mathrm{P}$ & 2 & Suhl-Matthias-Walker \\
\hline & $\bigoplus_{\mathbf{l}} s u(1,1)-\mathrm{B}$ & & Lipkin-Meshkov-Glick \\
\hline & & 2 & Interacting boson (IBM1) \\
\hline & & & Two-Josephson-coupled BECs \\
\hline & $\bigoplus_{\mathbf{l}} s u(2) \oplus h_{4}$ & $N$ & Generalized Dicke, F-atom-molecule \\
\hline & $\bigoplus_{\mathbf{l}} s u(1,1) \oplus h_{4}$ & $N$ & B-atom-molecule \\
\hline & $\bigoplus_{\mathbf{l}} s u(2)-\mathrm{F}-\mathrm{S} \oplus s u(2)$ & $N$ & Kondo-like impurity \\
\hline & $\bigoplus_{\mathbf{l}} h_{4} \oplus s u(2)$ & $N$ & Special spin-boson \\
\hline$X Y Z$ & $\bigoplus_{\mathbf{l}} \operatorname{su}(2)$ & $N$ & Generalized $X Y Z$ Gaudin \\
\hline
\end{tabular}

In Section 4 we consider two possible realizations of the GGA in terms of the generators of $\bigoplus_{\mathrm{I}} s u(2)$ and $\bigoplus_{\mathrm{I}} s u(1,1)$ which allow us to construct (given the analytic properties of the solutions of the Gaudin equation) general Gaudin model Hamiltonians that will be exploited in the rest of the paper. Clearly, from the oscillator realizations of $\bigoplus_{\mathbf{l}} s u(2)$ and $\bigoplus_{\mathrm{I}} s u(1,1)$ in terms of canonical fermions and bosons, one can build several interesting many-body Hamiltonians, including the BCS, SMW, LMG, and GD. But one is not limited to these oscillators realizations. Indeed, one can use, for instance, $S U(N)$ or hard-core particles realizations to construct new exactly-solvable Hamiltonians [12].

Sections 5 and 6 present applications of the algebraic framework to various well-known models. They correspond to different realizations of the algebras $s u(2)$ or $s u(1,1)$ in terms of canonical fermions or bosons (see Table 1). We start Section 5 by solving the BCS pairing models in an arbitrary basis and then focus on the analysis of the BCS Hamiltonian in momentum space. We study, particularly, multiband pairing Hamiltonians such as the SMW model which is of relevance for the description of two-gap superconductivity in $\mathrm{MgB}_{2}$. In Section 6, we analyze bosonic pairing models of interest in cold atom physics. In particular, Section 6.3 concentrates on exactly-solvable two-level boson Hamiltonians, which include the nuclear interacting boson model (IBM) [13], the two Josephson-coupled Bose-Einstein condensates (BECs) model [14], and the LMG model. Most importantly, we show that the LMG model, used for decades to study phase transitions in finite nuclei, is exactly solvable.

One would like to understand what are the general differences between mean-field approximations to the $X Y Z$ Gaudin models, and their exact solution. As we will see, for finite 
systems the distinction is evident and the character of the solutions of the two approaches differs substantially. However, does the difference persist in the thermodynamic limit, i.e., the infinite $(N \rightarrow \infty)$ system-size limit? Expectation values of certain observables (e.g., the BCS gap equation or the occupation numbers) will be identical, but other observables may pick up the differences. A question that naturally arises concerns the critical behavior of the $X Y Z$ Gaudin models. One would like to know, for example, what are their quantum critical exponents. It turns out that for certain Gaudin models (e.g., the LMG and SMW model of Eq. (71)) the critical behavior is mean-field [15]. This is very simple to prove by applying tools from Lie algebras and catastrophe theory, as developed by Gilmore [16]. The general analysis is beyond the scope of the present paper and will be presented in a separate publication. Here, however, we will only analyze the quantum phase diagram of the BCS model as a function of the interaction strength and show that the transition between the superconducting and Fermi-liquid phases is Kosterlitz-Thouless-like [17], independently of the space dimensionality of the lattice.

Mixing realizations and representations of the generators of the Gaudin algebra lead to new exactly-solvable models (Table 1). In Section 7 we illustrate these ideas by solving three types of many-body models: the GD, an exactly-solvable Kondo-like, and a spin-boson models. In this way, one finds the formal algebraic connection between these different physical phenomena and BCS superconductivity. Section 8 deals with differential operator realizations of the Gaudin generators leading to quasiexactly solvable problems in the continuum. Finally, we show in Appendix A that the weak-coupling limit solutions of the generalized $X X Z$ Gaudin models are given by the roots of Laguerre polynomials.

\section{Generalized Gaudin algebras}

\subsection{Commutation relations}

Let us introduce the GGA as the set of operators $\left\{\mathrm{S}_{m}^{\kappa} \equiv \mathrm{S}^{\kappa}\left(E_{m}\right)\right\}$, with $\kappa=x, y, z$, satisfying the commutation relations (for $E_{m} \neq E_{\ell}$ )

$$
\left\{\begin{array}{l}
{\left[\mathrm{S}_{m}^{\kappa}, \mathrm{S}_{\ell}^{\kappa}\right]=0} \\
{\left[\mathrm{~S}_{m}^{x}, \mathrm{~S}_{\ell}^{y}\right]=i\left(Y_{m \ell} \mathrm{S}_{m}^{z}-X_{m \ell} \mathrm{S}_{\ell}^{z}\right)} \\
{\left[\mathrm{S}_{m}^{y}, \mathrm{~S}_{\ell}^{z}\right]=i\left(Z_{m \ell} \mathrm{S}_{m}^{x}-Y_{m \ell} \mathrm{S}_{\ell}^{x}\right)} \\
{\left[\mathrm{S}_{m}^{z}, \mathrm{~S}_{\ell}^{x}\right]=i\left(X_{m \ell} \mathrm{S}_{m}^{y}-Z_{m \ell} \mathrm{S}_{\ell}^{y}\right)}
\end{array}\right.
$$

where $X_{m \ell}=X\left(E_{m}, E_{\ell}\right), Y_{m \ell}=Y\left(E_{m}, E_{\ell}\right)$, and $Z_{m \ell}=Z\left(E_{m}, E_{\ell}\right)$ are antisymmetric (i.e., $W(x, y)=-W(y, x))$ complex functions of two arbitrary complex variables $E_{m}, E_{\ell}$ labelled by positive integers $m$ and $\ell$, respectively. Equivalently, in terms of the $\kappa=+,-, z$ basis (and for $E_{m} \neq E_{\ell}$ )

$$
\left\{\begin{array}{l}
{\left[\mathrm{S}_{m}^{ \pm}, \mathrm{S}_{\ell}^{ \pm}\right]= \pm 2 V_{m \ell}^{-}\left(\mathrm{S}_{m}^{z}+\mathrm{S}_{\ell}^{z}\right)} \\
{\left[\mathrm{S}_{m}^{-}, \mathrm{S}_{\ell}^{+}\right]=-2 V_{m \ell}^{+}\left(\mathrm{S}_{m}^{z}-\mathrm{S}_{\ell}^{z}\right)} \\
{\left[\mathrm{S}_{m}^{z}, \mathrm{~S}_{\ell}^{ \pm}\right]= \pm\left(V_{m \ell}^{+} \mathrm{S}_{m}^{ \pm}-Z_{m \ell} \mathrm{S}_{\ell}^{ \pm}-V_{m \ell}^{-} \mathrm{S}_{m}^{\mp}\right)}
\end{array}\right.
$$


where $\mathrm{S}_{m}^{ \pm}=\mathrm{S}_{m}^{x} \pm i \mathrm{~S}_{m}^{y}$, and $V_{m \ell}^{ \pm}=\left(X_{m \ell} \pm Y_{m \ell}\right) / 2$. (Notice that $\mathrm{S}_{m}^{+(-)}$and $\mathrm{S}_{\ell}^{+(-)}$are non-commuting operators, unless $X_{m \ell}=Y_{m \ell}$.)

The complex functions $X_{m \ell}, Y_{m \ell}$, and $Z_{m \ell}$ are taken to have the limiting behavior

$$
\begin{array}{ll}
\lim _{\varepsilon \rightarrow 0} \varepsilon X(x, x+\varepsilon)=\mathrm{f}(x), & \lim _{\varepsilon \rightarrow 0} \varepsilon Y(x, x+\varepsilon)=\mathrm{g}(x), \\
\lim _{\varepsilon \rightarrow 0} \varepsilon Z(x, x+\varepsilon)=\mathrm{h}(x), &
\end{array}
$$

where $\mathrm{f}(x), \mathrm{g}(x)$, and $\mathrm{h}(x)$ are nonsingular functions. Indeed, $X, Y$, and $Z$ are complex meromorphic functions having poles of order one. In particular, when $\mathrm{f}(x)=\mathrm{g}(x)=\mathrm{h}(x)$ the above commutation relations, Eqs. (1), can be analytically continued to the case $m=\ell$ (i.e., $E_{m} \rightarrow E_{\ell}$ ). For example,

$$
\begin{aligned}
{\left[\mathrm{S}_{m}^{x}, \mathrm{~S}_{m}^{y}\right] } & =\lim _{\varepsilon \rightarrow 0} i\left(Y\left(E_{m}, E_{m}+\varepsilon\right) \mathrm{S}^{z}\left(E_{m}\right)-X\left(E_{m}, E_{m}+\varepsilon\right) \mathrm{S}^{z}\left(E_{m}+\varepsilon\right)\right) \\
& =-i \mathrm{f}\left(E_{m}\right) \frac{\partial \mathrm{S}_{m}^{z}}{\partial E_{m}} .
\end{aligned}
$$

Then,

$$
\left\{\begin{array}{l}
{\left[\mathrm{S}_{m}^{\kappa}, \mathrm{S}_{m}^{\kappa}\right]=0,} \\
{\left[\mathrm{~S}_{m}^{x}, \mathrm{~S}_{m}^{y}\right]=-i \mathrm{f}\left(E_{m}\right) \frac{\partial \mathrm{S}_{m}^{z}}{\partial E_{m}},} \\
{\left[\mathrm{~S}_{m}^{y}, \mathrm{~S}_{m}^{z}\right]=-i \mathrm{f}\left(E_{m}\right) \frac{\partial \mathrm{S}_{m}^{x}}{\partial E_{m}},} \\
{\left[\mathrm{~S}_{m}^{z}, \mathrm{~S}_{m}^{x}\right]=-i \mathrm{f}\left(E_{m}\right) \frac{\partial \mathrm{S}_{m}^{y}}{\partial E_{m}},}
\end{array}\right.
$$

which together with Eqs. (1) form an infinite-dimensional Lie algebra.

From the Jacobi identities for the generators of this Lie algebra, for example

$$
\left[\mathrm{S}_{n}^{x},\left[\mathrm{~S}_{m}^{x}, \mathrm{~S}_{\ell}^{y}\right]\right]+\left[\mathrm{S}_{\ell}^{y},\left[\mathrm{~S}_{n}^{x}, \mathrm{~S}_{m}^{x}\right]\right]+\left[\mathrm{S}_{m}^{x},\left[\mathrm{~S}_{\ell}^{y}, \mathrm{~S}_{n}^{x}\right]\right]=0
$$

we obtain, considering the antisymmetry of the functions $X, Y, Z$, the Gaudin equations [19]

$$
Z_{m \ell} X_{\ell n}+Z_{n m} Y_{\ell n}+X_{n m} Y_{m \ell}=0 .
$$

Moreover, the relations (for any pair of indices $m, \ell$ )

$$
X_{m \ell}^{2}-Z_{m \ell}^{2}=\Gamma_{1}, \quad X_{m \ell}^{2}-Y_{m \ell}^{2}=\Gamma_{2}
$$

also result from these identities, where $\Gamma_{1,2}$ are constants independent of $E_{m}$ and $E_{\ell}$.

\subsection{Quantum invariants}

Let us introduce the generalized Gaudin field operators

$$
H\left(E_{m}\right) \equiv H_{m}=\mathrm{S}_{m}^{x} \mathrm{~S}_{m}^{x}+\mathrm{S}_{m}^{y} \mathrm{~S}_{m}^{y}+\mathrm{S}_{m}^{z} \mathrm{~S}_{m}^{z}=\frac{1}{2}\left(\mathrm{~S}_{m}^{+} \mathrm{S}_{m}^{-}+\mathrm{S}_{m}^{-} \mathrm{S}_{m}^{+}+2 \mathrm{~S}_{m}^{z} \mathrm{~S}_{m}^{z}\right)
$$


which act on a carrier space $\mathcal{H}$. These operators are not the Casimir operators of the GGA since they do not commute with its generators $\left(E_{m} \neq E_{\ell}\right)$

$$
\left\{\begin{array}{l}
{\left[H_{m}, \mathrm{~S}_{\ell}^{ \pm}\right]= \pm\left(V_{m \ell}^{+}\left\{\mathrm{S}_{m}^{ \pm}, \mathrm{S}_{\ell}^{z}\right\}+V_{m \ell}^{-}\left\{\mathrm{S}_{m}^{\mp}, \mathrm{S}_{\ell}^{z}\right\}-Z_{m \ell}\left\{\mathrm{S}_{\ell}^{ \pm}, \mathrm{S}_{m}^{z}\right\}\right),} \\
{\left[H_{m}, \mathrm{~S}_{\ell}^{z}\right]=V_{m \ell}^{+}\left(\mathrm{S}_{\ell}^{+} \mathrm{S}_{m}^{-}-\mathrm{S}_{m}^{+} \mathrm{S}_{\ell}^{-}\right)+V_{m \ell}^{-}\left(\mathrm{S}_{\ell}^{+} \mathrm{S}_{m}^{+}-\mathrm{S}_{m}^{-} \mathrm{S}_{\ell}^{-}\right),}
\end{array}\right.
$$

where $\{\hat{A}, \hat{B}\}=\hat{A} \hat{B}+\hat{B} \hat{A}$ is the anticommutator. A key property is that these field operators form a commutative family

$$
\left[H_{m}, H_{\ell}\right]=0,
$$

therefore, they have a common set of eigenvectors in $\mathcal{H}$ and consequently can be considered as a generating function for all conserved quantities of quantum integrable systems which will be called generalized $X Y Z$ Gaudin models.

\section{The $X X Z$ Gaudin models}

In the following we will concentrate on the diagonalization of the $X X Z$ Gaudin models, i.e., the cases where $X_{m \ell}=Y_{m \ell}$ [18]. As we will see in the applications, this is the most relevant case from a physics standpoint, and the simpler mathematically since the CartanWeyl basis is easily defined. The generalized $X Y Z$ models will be analyzed somewhere else.

\subsection{Solutions of the $X X Z$ Gaudin equation}

The Gaudin equation (6) reduces to

$$
Z_{m \ell} X_{\ell n}+Z_{n m} X_{\ell n}+X_{n m} X_{m \ell}=0 .
$$

From this expression, together with the antisymmetry of the functions $X$ and $Z$, one can derive a parametrization for the coefficients $Z_{\ell n}$ and $X_{\ell n}$ :

$$
X_{\ell n}=\frac{X_{m \ell} X_{m n}}{Z_{m \ell}-Z_{m n}}, \quad Z_{\ell n}=\frac{Z_{m n} Z_{m \ell}+X_{m n}^{2}-Z_{m n}^{2}}{Z_{m \ell}-Z_{m n}} .
$$

From the latter expression, and $Z_{\ell n}=-Z_{n \ell}$, it follows that

$$
X_{m n}^{2}-Z_{m n}^{2}=X_{m \ell}^{2}-Z_{m \ell}^{2}=\Gamma,
$$

with $\Gamma$ a constant that is independent of any indices. Taking $E_{r}$ as a reference parameter, one can write down

$$
X_{\ell n}=\frac{X_{r \ell} X_{r n}}{Z_{r \ell}-Z_{r n}}, \quad Z_{\ell n}=\frac{\Gamma+Z_{r n} Z_{r \ell}}{Z_{r \ell}-Z_{r n}} .
$$

Then, the functions $X_{\ell n}$ and $Z_{\ell n}$, which satisfy the Gaudin equations (10), can be written in terms of a limited set of parameters $s, g$, and $t_{i}$ as

$$
X_{\ell n}=g \frac{\sqrt{1+s t_{\ell}^{2}} \sqrt{1+s t_{n}^{2}}}{t_{\ell}-t_{n}}, \quad Z_{\ell n}=g \frac{1+s t_{\ell} t_{n}}{t_{\ell}-t_{n}},
$$


with

$$
\Gamma=s g^{2}, \quad t_{i}=-g / Z_{r i}
$$

where $g$ is a real number and $|s|=0$ or 1 . Taking the limit $t_{\ell} \rightarrow t_{n}=x$, one finds the limiting behavior defined in Eq. (3) as

$$
\mathrm{f}(x)=\mathrm{g}(x)=\mathrm{h}(x)=g\left(1+s x^{2}\right) .
$$

In all practical cases one can take the square roots in Eq. (14) to be real and positive (normally any phase can be absorbed in the definition of the generators $\mathrm{S}^{+}$and $\mathrm{S}^{-}$). Furthermore, the condition that the resulting exactly-solvable Hamiltonians should be Hermitian leads in most cases to the condition that the parameters $\Gamma$ and $t_{i}$ be real. In this case the parameter $s$ is either $+1,-1$ or 0 .

This corresponds to the three cases discussed by Gaudin [19]:

(1) Rational: $\Gamma=0, s=0$,

$$
X\left(\eta_{\ell}, \eta_{n}\right)=Z\left(\eta_{\ell}, \eta_{n}\right)=g \frac{1}{\eta_{\ell}-\eta_{n}},
$$

with $t_{i}=\eta_{i}$.

(2) Trigonometric: $\Gamma>0, s=+1$,

$$
X\left(\eta_{\ell}, \eta_{n}\right)=g \frac{1}{\sin \left(\eta_{\ell}-\eta_{n}\right)}, \quad Z\left(\eta_{\ell}, \eta_{n}\right)=g \cot \left(\eta_{\ell}-\eta_{n}\right)
$$

with $t_{i}=\tan \left(\eta_{i}\right)$.

(3) Hyperbolic: $\Gamma<0, s=-1$,

$$
X\left(\eta_{\ell}, \eta_{n}\right)=g \frac{1}{\sinh \left(\eta_{\ell}-\eta_{n}\right)}, \quad Z\left(\eta_{\ell}, \eta_{n}\right)=g \operatorname{coth}\left(\eta_{\ell}-\eta_{n}\right),
$$

with $t_{i}=\tanh \left(\eta_{i}\right)$.

Note that for these three parametrizations one finds that the limiting behavior is given by

$$
\mathrm{f}(x)=\mathrm{g}(x)=\mathrm{h}(x)=g,
$$

and that the rational model corresponds to the limit $\eta_{\ell} \rightarrow \eta_{n}$ of both the trigonometric and the hyperbolic model.

Recently, Richardson has proposed a new family of solutions, given by [11]

$$
\begin{aligned}
& X\left(z_{\ell}, z_{n}\right)=\frac{\sqrt{1+2 \alpha z_{\ell}+\beta z_{\ell}^{2}} \sqrt{1+2 \alpha z_{n}+\beta z_{n}^{2}}}{z_{\ell}-z_{n}}, \\
& Z\left(z_{\ell}, z_{n}\right)=\frac{1+\alpha\left(z_{\ell}+z_{n}\right)+\beta z_{\ell} z_{n}}{z_{\ell}-z_{n}} .
\end{aligned}
$$

Evaluating expression Eq. (12) for this parametrization, one finds that $\Gamma=\beta-\alpha^{2}$. Hence depending on the sign of $\beta-\alpha^{2}$, one finds that this solution might be expressed as a reparametrization of the rational, trigonometric or hyperbolic models. 
Another useful parametrization is given by

$$
X\left(\eta_{\ell}, \eta_{n}\right)=g \frac{2 c_{\ell} c_{n}}{c_{\ell}^{2}-c_{n}^{2}}, \quad Z\left(\eta_{\ell}, \eta_{n}\right)=g \frac{c_{\ell}^{2}+c_{n}^{2}}{c_{\ell}^{2}-c_{n}^{2}},
$$

which can be derived from the hyperbolic parametrization, Eq. (19), by taking $c_{i}=e^{\eta_{i}}$.

\subsection{Diagonalizing the $X X Z$ Gaudin models}

To define the representation (or carrier) space $\mathcal{H}$ of the generalized $X X Z$ GGA we introduce the lowest-weight vector $|0\rangle$, such that,

$$
\mathrm{S}_{m}^{-}|0\rangle=0, \quad \mathrm{~S}_{m}^{z}|0\rangle=F\left(E_{m}\right)|0\rangle \forall E_{m},
$$

with $F\left(E_{m}\right)$ the lowest-weight function. Thus, the carrier space $\mathcal{H}$ is defined as the linear span of the unnormalized vectors

$$
\left\{|0\rangle, S_{1}^{+}|0\rangle, S_{1}^{+} S_{2}^{+}|0\rangle, \ldots, S_{1}^{+} S_{2}^{+} \ldots S_{m}^{+}|0\rangle, \ldots\right\} .
$$

We want now to diagonalize the Gaudin field operators. Using Eqs. (4) it turns out that $|0\rangle$ is an eigenstate of $H_{m}$ with eigenvalue

$$
\omega_{0}\left(E_{m}\right)=F^{2}\left(E_{m}\right)-\mathrm{f}\left(E_{m}\right) \frac{\partial}{\partial E_{m}} F\left(E_{m}\right) .
$$

To solve the general eigenvalue problem

$$
H_{m}|\Phi\rangle=\omega\left(E_{m}\right)|\Phi\rangle,
$$

we propose the Bethe ansatz $\left(M \in \mathbb{Z}^{+}\right)$

$$
|\Phi\rangle=\prod_{\ell=1}^{M} \mathrm{~S}_{\ell}^{+}|0\rangle=\mathrm{S}_{1}^{+} \mathrm{S}_{2}^{+} \ldots \mathrm{S}_{M}^{+}|0\rangle
$$

and Eq. (26) is equivalent to

$$
\left(H_{m}-\omega_{0}\left(E_{m}\right)\right)|\Phi\rangle=\left[H_{m}, \prod_{\ell=1}^{M} \mathrm{~S}_{\ell}^{+}\right]|0\rangle .
$$

Thus, the whole problem reduces to compute the commutator

$$
\left[H_{m}, \prod_{\ell=1}^{M} \mathrm{~S}_{\ell}^{+}\right]=\sum_{\ell=1}^{M}\left(\left(\prod_{n=1}^{\ell-1} \mathrm{~S}_{n}^{+}\right)\left[H_{m}, \mathrm{~S}_{\ell}^{+}\right]\left(\prod_{n=\ell+1}^{M} \mathrm{~S}_{n}^{+}\right)\right)
$$

whose action upon the state $|0\rangle$ can be written as

$$
\begin{aligned}
{\left[H_{m}, \prod_{\ell=1}^{M} \mathrm{~S}_{\ell}^{+}\right]|0\rangle=} & \sum_{\ell=1}^{M}\left(\prod_{r(\neq \ell)}^{M} \mathrm{~S}_{r}^{+}\right)\left[H_{m}, \mathrm{~S}_{\ell}^{+}\right]|0\rangle \\
& +\frac{1}{2} \sum_{\ell \neq n=1}^{M}\left(\prod_{r(\neq \ell, n)}^{M} \mathrm{~S}_{r}^{+}\right)\left[\left[H_{m}, \mathrm{~S}_{\ell}^{+}\right], \mathrm{S}_{n}^{+}\right]|0\rangle
\end{aligned}
$$


which after some algebraic manipulations reduces to

$$
\begin{aligned}
& \sum_{\ell=1}^{M}\left(\Gamma-2 Z_{m \ell} F\left(E_{m}\right)+\sum_{n(\neq \ell)=1}^{M} Z_{m \ell} Z_{m n}\right)|\Phi\rangle \\
& \quad+2 \sum_{\ell=1}^{M}\left(X_{m \ell} F\left(E_{\ell}\right)+\sum_{n(\neq \ell)=1}^{M} X_{m \ell} Z_{n \ell}\right) \hat{\Psi}_{\ell m}^{+}|0\rangle,
\end{aligned}
$$

with $\hat{\Psi}_{\ell m}^{+}$given by

$$
\hat{\Psi}_{\ell m}^{+}=\left(\prod_{n=1}^{\ell-1} \mathrm{~S}_{n}^{+}\right) \mathrm{S}_{m}^{+}\left(\prod_{n=\ell+1}^{M} \mathrm{~S}_{n}^{+}\right)
$$

Equating to zero all the coefficients in front of $\hat{\Psi}_{\ell m}^{+}$, defines a set of nonlinear coupled equations

$$
F\left(E_{\ell}\right)+\sum_{n(\neq \ell)=1}^{M} Z_{n \ell}=0, \quad \ell=1, \ldots, M,
$$

termed Bethe's equations, which determine the set of complex numbers $\left\{E_{m}\right\}$. Once they are solved, one uses these solutions to write down the eigenvalues

$$
\omega\left(E_{m}\right)=\omega_{0}\left(E_{m}\right)+\sum_{\ell=1}^{M}\left(\Gamma-2 Z_{m \ell} F\left(E_{m}\right)+\sum_{n(\neq \ell)=1}^{M} Z_{m \ell} Z_{m n}\right) .
$$

\section{Exactly-solvable models derived from the Gaudin algebra}

Thus far, we have not assumed any special form for the generators of the GGA. Let us consider now a possible realization in terms of generators of the $\bigoplus_{\mathbf{l}} \operatorname{su}(2)=\operatorname{su}(2) \oplus$ $s u(2) \oplus \cdots \oplus s u(2)$ algebra, which satisfy the relations

$$
\left[S_{\mathbf{i}}^{+}, S_{\mathbf{j}}^{-}\right]=2 \delta_{\mathbf{i j}} S_{\mathbf{j}}^{z}, \quad\left[S_{\mathbf{i}}^{z}, S_{\mathbf{j}}^{ \pm}\right]= \pm \delta_{\mathbf{i j}} S_{\mathbf{j}}^{ \pm},
$$

with $\left(S_{\mathbf{j}}^{+}\right)^{\dagger}=S_{\mathbf{j}}^{-}$. The set of indices $\mathbf{j}$ will be denoted by the symbol $\mathcal{T}$, whose cardinal is $N_{\mathcal{T}}$. Defining the following operators in terms of the $\bigoplus_{\mathbf{l}} s u(2)$ generators,

$$
\mathrm{S}_{m}^{ \pm}=\sum_{\mathbf{j} \in \mathcal{T}} X_{m \mathbf{j}} S_{\mathbf{j}}^{ \pm}, \quad \mathrm{S}_{m}^{z}=-\frac{1}{2} \mathbb{1}-\sum_{\mathbf{j} \in \mathcal{T}} Z_{m \mathbf{j}} S_{\mathbf{j}}^{z},
$$

one has a possible realization of the generators of the GGA, Eq. (1).

In the $X Y Z$ case they are given by

$$
\mathrm{S}_{m}^{x}=\sum_{\mathbf{j} \in \mathcal{T}} X_{m \mathbf{j}} S_{\mathbf{j}}^{x}, \quad \mathrm{~S}_{m}^{y}=\sum_{\mathbf{j} \in \mathcal{T}} Y_{m \mathbf{j}} S_{\mathbf{j}}^{y}, \quad \mathrm{~S}_{m}^{z}=-\sum_{\mathbf{j} \in \mathcal{T}} Z_{m \mathbf{j}} S_{\mathbf{j}}^{z} .
$$


Notice that the $s u(2)$ generators are not constrained to be in any particular irreducible representation. In the $\mathrm{S}=1 / 2$ case, the elliptic $X Y Z$ Gaudin model has been shown to be exactly solvable $[20,21]$. Note that for arbitrary $S$ the model is still quantum integrable as shown in Eq. (9).

Similarly, one can realize the generators of the GGA in terms of the generators of the Lie algebra $\bigoplus_{\mathrm{l}} s u(1,1)$, homomorphic to $\bigoplus_{\mathrm{I}} s u(2)$, which satisfy

$$
\left[K_{\mathbf{i}}^{+}, K_{\mathbf{j}}^{-}\right]=-2 \delta_{\mathbf{i j}} K_{\mathbf{j}}^{z}, \quad\left[K_{\mathbf{i}}^{z}, K_{\mathbf{j}}^{ \pm}\right]= \pm \delta_{\mathbf{i j}} K_{\mathbf{j}}^{ \pm},
$$

with $\left(K_{\mathbf{j}}^{+}\right)^{\dagger}=K_{\mathbf{j}}^{-}$, obtaining

$$
\mathrm{S}_{m}^{+}=\sum_{\mathbf{j} \in \mathcal{T}} X_{m \mathbf{j}} K_{\mathbf{j}}^{+}, \quad \mathrm{S}_{m}^{-}=-\sum_{\mathbf{j} \in \mathcal{T}} X_{m \mathbf{j}} K_{\mathbf{j}}^{-}, \quad \mathrm{S}_{m}^{z}=-\frac{1}{2} \mathbb{1}-\sum_{\mathbf{j} \in \mathcal{T}} Z_{m \mathbf{j}} K_{\mathbf{j}}^{z} .
$$

For the sake of simplicity, we will proceed with the Gaudin operators defined from the $\bigoplus_{\mathrm{I}} s u(2)$ generators. Extension to $\bigoplus_{\mathrm{l}} s u(1,1)$ is straightforward after application of the non-unitary homomorphic mapping: $\mathrm{S}_{m}^{+} \rightarrow \mathrm{S}_{m}^{+}, \mathrm{S}_{m}^{-} \rightarrow-\mathrm{S}_{m}^{-}, \mathrm{S}_{m}^{z} \rightarrow \mathrm{S}_{m}^{z}$. It is easy to check that the Gaudin field operators, Eq. (8), are given by

$$
H_{m}=\sum_{\mathbf{i} \in \mathcal{T}} Z_{m \mathbf{i}} S_{\mathbf{i}}^{z}+\sum_{\mathbf{i}, \mathbf{j} \in \mathcal{T}}\left(Z_{m \mathbf{i}} Z_{m \mathbf{j}} S_{\mathbf{i}}^{z} S_{\mathbf{j}}^{z}+\frac{X_{m \mathbf{i}} X_{m \mathbf{j}}}{2}\left(S_{\mathbf{i}}^{+} S_{\mathbf{j}}^{-}+S_{\mathbf{i}}^{-} S_{\mathbf{j}}^{+}\right)\right)+\frac{1}{4},
$$

where we assume that $X_{m \mathbf{i}}=X\left(E_{m}, \eta_{\mathbf{i}}\right)$ and $Z_{m \mathbf{i}}=Z\left(E_{m}, \eta_{\mathbf{i}}\right)$. Bethe's equations, Eq. (33), are given by

$$
1+2 \sum_{\mathbf{j} \in \mathcal{T}} d_{\mathbf{j}} Z_{\ell \mathbf{j}}+2 \sum_{n(\neq \ell)=1}^{M} Z_{\ell n}=0, \quad \ell=1, \ldots, M,
$$

where $d_{\mathbf{j}}$ is the eigenvalue of $S_{\mathbf{j}}^{z}\left(K_{\mathbf{j}}^{z}\right)$, i.e., $S_{\mathbf{j}}^{z}\left(K_{\mathbf{j}}^{z}\right)|0\rangle=d_{\mathbf{j}}|0\rangle$. In the weakly interacting limit the solutions of these equations are given by the roots of associated Laguerre polynomials (see Appendix A). This establishes a one-to-one correspondence between the eigenstates of the non-interacting and the weakly-interacting models, which proves that the Bethe ansatz covers all eigenstates and does not contain any spurious solutions for finite values of $g$.

In the strong interaction limit, $g \rightarrow \pm \infty$, some of the variables diverge to infinity, where again they can be related to roots of the associated Laguerre polynomials, scaled with a factor $g$. However, some of the roots can remain finite. These finite roots are equivalent to the solutions for the Gaudin spin magnets [19] and can be related to the elementary excitations of the BCS-model in the canonical ensemble [22].

From the analytic properties of the $X$ and $Z$ function matrices $\left(\eta_{\mathbf{i}} \neq \eta_{\mathbf{j}}\right)$

$$
\begin{aligned}
& \oint_{\Gamma_{\mathbf{i}}} \frac{d E_{m}}{2 \pi i} X_{m \mathbf{i}} X_{m \mathbf{j}}=\mathrm{f}\left(\eta_{\mathbf{i}}\right) X_{\mathbf{i j}}, \quad \oint_{\Gamma_{\mathbf{i}}} \frac{d E_{m}}{2 \pi i} Z_{m \mathbf{i}} Z_{m \mathbf{j}}=\mathrm{f}\left(\eta_{\mathbf{i}}\right) Z_{\mathbf{i} \mathbf{j}}, \\
& \oint_{\Gamma_{\mathbf{i}}} \frac{d E_{m}}{2 \pi i} Z_{m \mathbf{i}}=\mathrm{f}\left(\eta_{\mathbf{i}}\right),
\end{aligned}
$$


where $\Gamma_{\mathbf{i}}$ is a contour in the complex- $E_{m}$ plane encircling $\eta_{\mathbf{i}}$. In this way, one can write down the constants of motion $R_{\mathbf{i}}=\frac{1}{\mathrm{f}\left(\eta_{\mathbf{i}}\right)} \oint_{\Gamma_{\mathbf{i}}} \frac{d E_{m}}{2 \pi i} H_{m},\left[R_{\mathbf{i}}, R_{\mathbf{j}}\right]=0$, as

$$
\begin{aligned}
& R_{\mathbf{i}}=S_{\mathbf{i}}^{z}+2 \sum_{\mathbf{j} \in \mathcal{T}(\neq \mathbf{i})}\left(\frac{X_{\mathbf{i} \mathbf{j}}}{2}\left(S_{\mathbf{i}}^{+} S_{\mathbf{j}}^{-}+S_{\mathbf{i}}^{-} S_{\mathbf{j}}^{+}\right)+Z_{\mathbf{i j}} S_{\mathbf{i}}^{z} S_{\mathbf{j}}^{z}\right), \\
& R_{\mathbf{i}}=K_{\mathbf{i}}^{z}-2 \sum_{\mathbf{j} \in \mathcal{T}(\neq \mathbf{i})}\left(\frac{X_{\mathbf{i j}}}{2}\left(K_{\mathbf{i}}^{+} K_{\mathbf{j}}^{-}+K_{\mathbf{i}}^{-} K_{\mathbf{j}}^{+}\right)-Z_{\mathbf{i j}} K_{\mathbf{i}}^{z} K_{\mathbf{j}}^{z}\right),
\end{aligned}
$$

for $\bigoplus_{\mathbf{I}} \operatorname{su}(2)$ and $\bigoplus_{\mathbf{I}} \operatorname{su}(1,1)$, respectively, with eigenvalues $r_{\mathbf{i}}=\frac{1}{\mathrm{f}\left(\eta_{\mathbf{i}}\right)} \oint_{\Gamma_{\mathbf{i}}} \frac{d E_{m}}{2 \pi i} \omega\left(E_{m}\right)$

$$
r_{\mathbf{i}}=d_{\mathbf{i}}\left(1+2 \sum_{\ell} Z_{\mathbf{i} \ell}+2 \sum_{\mathbf{j} \in \mathcal{T}(\neq \mathbf{i})} d_{\mathbf{j}} Z_{\mathbf{i j}}\right) .
$$

A class of Gaudin model Hamiltonians can be written as $H_{\mathrm{G}}=\sum_{\mathbf{i}} \varepsilon_{\mathbf{i}} R_{\mathbf{i}}$, i.e.,

$$
\begin{aligned}
& H_{\mathrm{G}}=\sum_{\mathbf{i}} \varepsilon_{\mathbf{i}} S_{\mathbf{i}}^{z}+\frac{\tilde{g}}{2 N} \sum_{\mathbf{i}, \mathbf{j}(\mathbf{i} \neq \mathbf{j})}\left(\tilde{X}_{\mathbf{i j}}\left(S_{\mathbf{i}}^{+} S_{\mathbf{j}}^{-}+S_{\mathbf{i}}^{-} S_{\mathbf{j}}^{+}\right)+2 \tilde{Z}_{\mathbf{i j}} S_{\mathbf{i}}^{z} S_{\mathbf{j}}^{z}\right), \\
& H_{\mathrm{G}}=\sum_{\mathbf{i}} \varepsilon_{\mathbf{i}} K_{\mathbf{i}}^{z}-\frac{\tilde{g}}{2 N} \sum_{\mathbf{i}, \mathbf{j}(\mathbf{i} \neq \mathbf{j})}\left(\tilde{X}_{\mathbf{i j}}\left(K_{\mathbf{i}}^{+} K_{\mathbf{j}}^{-}+K_{\mathbf{i}}^{-} K_{\mathbf{j}}^{+}\right)-2 \tilde{Z}_{\mathbf{i j}} K_{\mathbf{i}}^{z} K_{\mathbf{j}}^{z}\right),
\end{aligned}
$$

for $\bigoplus_{\mathbf{l}} s u(2)$ and $\bigoplus_{\mathbf{l}} s u(1,1)$, respectively. In the equations above $\varepsilon_{\mathbf{i}}$ is an arbitrary real number, $\tilde{X}_{\mathbf{i j}}=\left(\varepsilon_{\mathbf{i}}-\varepsilon_{\mathbf{j}}\right) X_{\mathbf{i j}} / g, \tilde{Z}_{\mathbf{i j}}=\left(\varepsilon_{\mathbf{i}}-\varepsilon_{\mathbf{j}}\right) Z_{\mathbf{i j}} / g$ are real symmetric matrix functions $\left(X_{\mathbf{i j}}=X\left(\eta_{\mathbf{i}}, \eta_{\mathbf{j}}\right), Z_{\mathbf{i j}}=Z\left(\eta_{\mathbf{i}}, \eta_{\mathbf{j}}\right)\right)$, and $\tilde{g}=g N$ is a $c$-number of order of magnitude unity because of thermodynamic stability reasons. Notice that, since $\varepsilon_{\mathbf{i}}$ and $\eta_{\mathbf{i}}$ are, in principle, independent parameters, one may take advantage of this freedom to write down different kinds of mode-dependent interactions (see Section 6.2). Moreover, $\varepsilon_{\mathbf{i}}$ and $\eta_{\mathbf{i}}$ must be chosen real for $H_{\mathrm{G}}$ to be Hermitian. Clearly, there are other classes of Gaudin models that involve higher-order combinations of the integrals of motion $R_{\mathbf{i}}$.

In the $X X X$ Gaudin models one can consider a more general representation of the Gaudin field operators in terms of the generators of $\bigoplus_{\mathrm{I}} \operatorname{su}(2)$ or $\bigoplus_{\mathrm{I}} \operatorname{su}(1,1)$

$$
\begin{aligned}
& \mathrm{S}_{m}^{x}=\frac{B_{x}}{2} \mathbb{1}+\sum_{\mathbf{j} \in \mathcal{T}} X_{m \mathbf{j}} S_{\mathbf{j}}^{x}, \quad \mathrm{~S}_{m}^{y}=\frac{B_{y}}{2} \mathbb{1}+\sum_{\mathbf{j} \in \mathcal{T}} X_{m \mathbf{j}} S_{\mathbf{j}}^{y}, \\
& \mathrm{~S}_{m}^{z}=-\frac{B_{z}}{2} \mathbb{1}-\sum_{\mathbf{j} \in \mathcal{T}} X_{m \mathbf{j}} S_{\mathbf{j}}^{z}, \\
& \mathrm{~S}_{m}^{x}=i\left(-\frac{B_{y}}{2} \mathbb{1}+\sum_{\mathbf{j} \in \mathcal{T}} X_{m \mathbf{j}} K_{\mathbf{j}}^{y}\right), \quad \mathrm{S}_{m}^{y}=i\left(\frac{B_{x}}{2} \mathbb{1}-\sum_{\mathbf{j} \in \mathcal{T}} X_{m \mathbf{j}} K_{\mathbf{j}}^{x}\right), \\
& \mathrm{S}_{m}^{z}=-\frac{B_{z}}{2} \mathbb{1}-\sum_{\mathbf{j} \in \mathcal{T}} X_{m \mathbf{j}} K_{\mathbf{j}}^{z},
\end{aligned}
$$


leading to the constants of motion $\left(\mathbf{B}=\left(B_{x}, B_{y}, B_{z}\right)\right.$ is a vector with components that are arbitrary $c$-numbers)

$$
\begin{aligned}
& R_{\mathbf{i}}=\mathbf{B} \cdot S_{\mathbf{i}}+2 \sum_{\mathbf{j} \in \mathcal{T}(\neq \mathbf{i})} X_{\mathbf{i j}} S_{\mathbf{i}} \cdot S_{\mathbf{j}}, \\
& R_{\mathbf{i}}=\mathbf{B} \cdot K_{\mathbf{i}}-\sum_{\mathbf{j} \in \mathcal{T}(\neq \mathbf{i})} X_{\mathbf{i j}}\left(K_{\mathbf{i}}^{+} K_{\mathbf{j}}^{-}+K_{\mathbf{i}}^{-} K_{\mathbf{j}}^{+}-2 K_{\mathbf{i}}^{z} K_{\mathbf{j}}^{z}\right),
\end{aligned}
$$

from which new Gaudin model Hamiltonians can be realized (e.g., central spin models).

Linear combinations of the $R_{\mathrm{i}}$ 's will be used in the next sections to derive different exactly-solvable model Hamiltonians. Different models result from using different realizations of $s u(2)$ (or $s u(1,1)$ ). In the following we will use (canonical) fermion and boson realizations, though, one could have used many others [12], such as $S U(N)$ spins or hard-core particles, leading to new exactly-solvable problems all of them having the same algebraic root. For example, for $S U(2)$ in the spin $S=1$ irreducible representation one can write down Eq. (46) (for the rational case) in terms of $s u(3)$ generators $\mathcal{S}^{\mu \nu}(\mu, v=0,1,2)$ in the fundamental representation as [12]

$$
H_{\mathrm{G}}=\sum_{\mathbf{i}} \varepsilon_{\mathbf{i}}\left(\mathcal{S}_{\mathbf{i}}^{11}-\mathcal{S}_{\mathbf{i}}^{22}\right)+\sum_{\mathbf{i}, \mathbf{j}(\mathbf{i} \neq \mathbf{j})} J_{\mathbf{i} \mathbf{j}}\left(\mathcal{S}_{\mathbf{i}}^{\mu \nu} \mathcal{S}_{\mathbf{j}}^{\nu \mu}-\mathcal{S}_{\mathbf{i}}^{\mu \nu} \tilde{\mathcal{S}}_{\mathbf{j}}^{\nu \mu}\right),
$$

where $J_{\mathbf{i j}}=\left(\varepsilon_{\mathbf{i}}-\varepsilon_{\mathbf{j}}\right) X_{\mathbf{i j}} / 2$, and $\tilde{\mathcal{S}}^{\nu \mu}$ are the generators of $s u(3)$ in the conjugate representation.

\section{5. $\bigoplus_{\mathrm{l}} s u(2)$ fermionic representation models}

\subsection{BCS-like models}

Not many models in condensed matter physics have attracted that much attention as the Bardeen-Cooper-Schrieffer (BCS) model of superconductivity [5], a remarkable phenomenon discovered in 1911 by Gilles Holst and Kamerlingh Onnes [23], and which is characterized by vanishing electrical resistance and perfect diamagnetism [24]. Soon after the introduction of the BCS model in condensed matter, Bohr, Mottelson and Pines [25] applied the BCS theory to the description of pairing correlations in finite nuclei. The BCS or pairing Hamiltonian is given by

$$
H_{\mathrm{BCS}}=\sum_{\mathbf{l}} \varepsilon_{\mathbf{l}} n_{\mathbf{l}}+\sum_{\mathbf{l} \sigma \mathbf{l}^{\prime} \sigma^{\prime}} g_{\mathbf{I} \mathbf{I}^{\prime}}^{\sigma \sigma^{\prime}} c_{\mathbf{l} \sigma}^{\dagger} c c_{\mathbf{l} \sigma}^{\dagger} c_{\overline{\mathbf{I}^{\prime} \sigma^{\prime}}} c_{\mathbf{I}^{\prime} \sigma^{\prime}}
$$

The operator $c_{\mathbf{l} \sigma}^{\dagger}\left(c_{\mathbf{l} \sigma}\right)$ creates (destroys) a fermion in the state $\mathbf{l} \sigma$, where $\sigma$ is the third projection of the internal spin degree of freedom S, I refers to all other quantum numbers needed to specify completely the state, and $n_{\mathbf{l}}=\sum_{\sigma} c_{\mathbf{l} \sigma}^{\dagger} c_{\mathbf{l} \sigma}$ is a number operator. Though, in principle, the state $\overline{\mathbf{l} \sigma}$ could be an arbitrary conjugate state to $\mathbf{l} \sigma$ (only a bijective relation between conjugate pairs is required), we will restrict here to time-reversal conjugate pairs. Under time-reversal (effected by an antiunitary operator) the position operator stays 
unchanged while the (linear or angular) momentum or spin operators change sign. Thus, the time-reversal transformation of single-particle states is specific to the choice of basis. For example, the eigenstates of a generic angular momentum operator $\mathbf{J}$, labelled as $|j m\rangle$, transform as

$$
|\overline{j m}\rangle=(-1)^{j-m}|j-m\rangle .
$$

Similarly, the time-reversal transformation of an annihilation operator in a basis of spin and linear momentum is $c_{\overline{\mathbf{k} \sigma}}=(-1)^{\mathrm{S}-\sigma} c_{-\mathbf{k}-\sigma}$, while for a basis of spin and position $c_{\overline{\mathbf{r}} \sigma}=c_{\mathbf{r} \bar{\sigma}}=(-1)^{\mathrm{S}-\sigma} c_{\mathbf{r}-\sigma}$. For the sake of clarity, we will assume a position basis in the following such that the time-reversal operation will be referred exclusively to the internal spin part of the states, i.e., $c_{\overline{\mathbf{l}} \bar{\sigma}}=c_{\mathbf{l} \bar{\sigma}}$.

The pairing Hamiltonian (53) with uniform couplings $g_{\mathbf{I I}^{\prime}}^{\sigma \sigma^{\prime}}=g / 4$ has been solved exactly in full generality by Richardson in a series of papers in the sixties [26]. This important development escaped the attention of the condensed matter and nuclear physics communities until very recently, when the Richardson's works were rediscovered in the study of ultrasmall superconducting grains. In order to regain the exact solution we will now present a specific representation of the $s u(2)$ generators in terms of fermions

$$
\tau_{\mathbf{l}}^{+}=\frac{1}{2} \sum_{\sigma} c_{\mathbf{l} \sigma}^{\dagger} c_{\mathbf{l} \bar{\sigma}}^{\dagger}=\left(\tau_{\mathbf{l}}^{-}\right)^{\dagger}, \quad \tau_{\mathbf{l}}^{z}=\frac{1}{2} \sum_{\sigma} c_{\mathbf{l} \sigma}^{\dagger} c_{\mathbf{l} \sigma}-\frac{1}{4} \Omega_{\mathbf{l}},
$$

where the operator $\tau_{\mathbf{l}}^{+}$creates a pair of fermions in time-reversal states and $\Omega_{\mathbf{l}}=2 \tau_{\mathbf{l}}+1$ is the degeneracy of the state $\mathbf{l}$ related to the pseudospin of the state $\mathbf{l}$. It can be readily verified that the three operators $\left\{\tau_{1}^{ \pm}, \tau_{1}^{z}\right\}$ satisfy the $s u(2)$ algebra (35).

The integrability of the BCS Hamiltonian (53) was recently demonstrated [27]. It was shown that $H_{\mathrm{BCS}}$ can be written as a linear combination of the integrals of motion of the rational family with $X_{\mathbf{i j}}=Z_{\mathbf{i j}}=g /\left(\varepsilon_{\mathbf{i}}-\varepsilon_{\mathbf{j}}\right)(17)$

$$
H_{\mathrm{BCS}}=\sum_{\mathbf{l}} \varepsilon_{\mathbf{l}} R_{\mathbf{l}}+C=\sum_{\mathbf{l}} \varepsilon_{\mathbf{l}}\left(2 \tau_{\mathbf{l}}^{z}+\frac{1}{2} \Omega_{\mathbf{l}}\right)+g \sum_{\mathbf{I I}^{\prime}} \tau_{\mathbf{l}^{+}}^{+} \tau_{\mathbf{I}^{\prime}}^{-} .
$$

The complete set of eigenstates of the pairing Hamiltonian are given by the product wavefunction

$$
|\Psi\rangle=\prod_{m=1}^{M} \mathrm{~S}_{m}^{+}|\nu\rangle, \quad \mathrm{S}_{m}^{+}=\sum_{\mathbf{l}} X_{m \mathbf{l}} \tau_{\mathbf{l}}^{+}=\sum_{\mathbf{l}} \frac{1}{E_{m}-2 \varepsilon_{\mathbf{l}}} \tau_{\mathbf{l}}^{+},
$$

where $|v\rangle \equiv\left|v_{1}, v_{2}, \ldots, v_{L}\right\rangle$, with $L$ the total number of single particle states, is a state of $v$ unpaired fermions $\left(v=\sum_{\mathbf{l}} v_{\mathbf{l}}\right)$ defined by

$$
\tau_{1}^{-}|\nu\rangle=0, \quad n_{1}|\nu\rangle=v_{\mathbf{l}}|v\rangle .
$$

The quantum numbers $\nu_{\mathbf{I}}$ are often referred to as Seniority quantum numbers in the nuclear physics literature.

The total number of particles is $N=2 M+v$, with $M$ the number of Cooper pairs. Each eigenstate (57) is completely defined by a set of $M$ spectral parameters (pair energies) $E_{m}$ 
which are a particular solution of the Richardson's equations

$$
1+\frac{g}{2} \sum_{\mathbf{l}=1}^{L} \frac{\Omega_{\mathbf{l}}-2 \nu_{\mathbf{l}}}{2 \varepsilon_{\mathbf{l}}-E_{m}}+2 g \sum_{\ell(\neq m)=1}^{M} \frac{1}{E_{m}-E_{\ell}}=0 .
$$

The eigenvalues of the BCS Hamiltonian are

$$
E=\sum_{\mathbf{l}=1}^{L} \varepsilon_{\mathbf{l}} v_{\mathbf{l}}+\sum_{m=1}^{M} E_{m} .
$$

One can easily relate the spectra of the repulsive $(g>0)$ and attractive $(g<0)$ cases: if one performs the following canonical particle-hole transformation

$$
\left\{\begin{array}{l}
c_{\mathbf{l} \sigma}^{\dagger} \rightarrow c_{\mathbf{l} \sigma}, \\
c_{\mathbf{l} \sigma} \rightarrow c_{\mathbf{l} \sigma}^{\dagger},
\end{array}\right.
$$

which is not a symmetry (although the interaction term is invariant), $H_{\mathrm{BCs}}(g)$ transforms as

$$
H_{\mathrm{BCS}}(g) \rightarrow \sum_{\mathbf{l}} \Omega_{\mathbf{l}} \varepsilon_{\mathbf{l}}-H_{\mathrm{BCS}}(-g),
$$

indicating the relation between the two spectra.

In recent years, the exact solution of the BCS Hamiltonian has been recovered in the study of ultrasmall superconducting (for a review see [28]). The specific Hamiltonian for grains assumes a set of $L$ equally spaced doubly-degenerate single particle states. Implying that $\Omega_{\mathbf{l}}=2$ and $\varepsilon_{\mathbf{l}}=\mathbf{l}$, with $\mathbf{l}=1,2, \ldots, L$. The Richardson's equations (58) reduce to

$$
1+g \sum_{\mathbf{l}=1}^{L} \frac{1-v_{\mathbf{l}}}{2 \varepsilon_{l}-E_{m}}+2 g \sum_{\ell(\neq m)=1}^{M} \frac{1}{E_{m}-E_{\ell}}=0,
$$

with $\nu_{\mathbf{I}}=0,1$. The ground state for an even number of particles $N$ is in the sector of no broken pairs, $v_{\mathbf{I}}=0$ for all $\mathbf{l}$, while for odd $N, v_{\mathbf{I}}=1$ for $\mathbf{l}=(N+1) / 2$ and zero otherwise. In other words, the Fermi level is blocked by a single particle, excluding it from the active space as can be seen from the second term in Eq. (62). The additional gap at the Fermi energy due to the blocking of this level is at the origin of the odd-even difference observed in the tunnelling spectra of small grains. The excited states of the model are either collective states (pairing vibrations) within the same Seniority subspace [22], or noncollective broken pairs [29].

While this pairing model has found great success describing the physics of ultrasmall grains, it can be likewise applied to axially deformed nuclei with nonequally spaced single particle levels. The reason that prevented its use in standard nuclear structure calculations for so many years, was the lack of an efficient numerical procedure to solve Eq. (62) for a large number of nonequally spaced levels. While in the equally-spaced case the method proposed by Richardson [30] allowed the treatment of systems with $\sim 10^{3}$ particles [31], the singularities arising in the numerical solutions of the equations with nonequally space levels are difficult to treat. Recently, it has been proposed a new numerical procedure to 
avoid the singularities which seems to be very promising [32], and it might open the scope for applications to several quantum systems.

The original BCS model for superconductivity [5] was introduced in the context of bulk metallic superconductors. In this case, electrons (spin- $1 / 2$ fermions) are confined in an arbitrary dimensional box with periodic boundary conditions with single-particle states of the Bloch type. Pairing occurs in momentum k-space. For pairing in the singlet $s$-wave channel and Cooper pairs with zero momentum $(\mathbf{k} \uparrow,-\mathbf{k} \downarrow)$ the BCS Hamiltonian can be written as $\left(n_{\mathbf{k} \sigma}=c_{\mathbf{k} \sigma}^{\dagger} c_{\mathbf{k} \sigma}\right.$ with $\left.\sigma=\uparrow, \downarrow\right)$

$$
\begin{aligned}
H_{\mathrm{BCS}} & =\sum_{\mathbf{k} \sigma} \varepsilon_{\mathbf{k} \sigma} n_{\mathbf{k} \sigma}+g \sum_{\mathbf{k} \mathbf{k}^{\prime}}^{\prime} c_{\mathbf{k} \uparrow}^{\dagger} c_{-\mathbf{k} \downarrow}^{\dagger} c_{-\mathbf{k}^{\prime} \downarrow} c_{\mathbf{k}^{\prime} \uparrow} \\
& =\sum_{\mathbf{k}}\left[\varepsilon_{\mathbf{k}}\left(n_{\mathbf{k} \uparrow}+n_{-\mathbf{k} \downarrow}\right)-g n_{\mathbf{k} \uparrow} n_{-\mathbf{k} \downarrow}\right]+g \sum_{\mathbf{k} \mathbf{k}^{\prime}} c_{\mathbf{k} \uparrow}^{\dagger} c_{-\mathbf{k} \downarrow}^{\dagger} c_{-\mathbf{k}^{\prime} \downarrow} c_{\mathbf{k}^{\prime} \uparrow},
\end{aligned}
$$

where the prime in the first double sum means that the terms $\mathbf{k}=\mathbf{k}^{\prime}$ are omitted, and $c_{\mathbf{k} \sigma}^{\dagger}$ creates an electron with momentum $\mathbf{k}$ and spin $\sigma$. It has been assumed time-reversal invariance, i.e., $\varepsilon_{\mathbf{k} \uparrow}=\varepsilon_{-\mathbf{k} \downarrow}=\varepsilon_{\mathbf{k}}$. The relevant $\operatorname{su}(2)$ algebra in this case is

$$
\tau_{\mathbf{k}}^{+}=c_{\mathbf{k} \uparrow}^{\dagger} c_{-\mathbf{k} \downarrow}^{\dagger}=\left(\tau_{\mathbf{k}}^{-}\right)^{\dagger}, \quad \tau_{\mathbf{k}}^{z}=\frac{1}{2}\left(n_{\mathbf{k} \uparrow}+n_{-\mathbf{k} \downarrow}-1\right),
$$

where $\Omega_{\mathbf{k}}=2$, i.e., the single particle states $\mathbf{k} \uparrow$ and $-\mathbf{k} \downarrow$ are degenerate. However, $\varepsilon_{\mathbf{k}}$ may, in principle, differ from $\varepsilon_{-\mathbf{k}}$. The Hamiltonians of Eqs. (63) and (56) are dynamically equivalent. To see this let us rewrite Eq. (63) in terms of the pseudospin operators $\tau$

$$
H_{\mathrm{BCS}}=\sum_{\mathbf{k}}\left[\varepsilon_{\mathbf{k}}\left(2 \tau_{\mathbf{k}}^{z}+1\right)-g\left(\tau_{\mathbf{k}}^{z}+2\left(\tau_{\mathbf{k}}^{z}\right)^{2}\right)\right]+g \sum_{\mathbf{k} \mathbf{k}^{\prime}} \tau_{\mathbf{k}}^{+} \tau_{\mathbf{k}^{\prime}}^{-} .
$$

It can be easily shown that the operators $\sum_{\mathbf{k}} \tau_{\mathbf{k}}^{z}$, and $\sum_{\mathbf{k}}\left(\tau_{\mathbf{k}}^{z}\right)^{2}$ are conserved quantities, i.e., commute with $H_{\mathrm{BCS}}$. Thus, up to an irrelevant global constant,

$$
H_{\mathrm{BCS}}=\sum_{\mathbf{k}} \varepsilon_{\mathbf{k}}\left(2 \tau_{\mathbf{k}}^{z}+1\right)+g \sum_{\mathbf{k} \mathbf{k}^{\prime}} \tau_{\mathbf{k}}^{+} \tau_{\mathbf{k}^{\prime}}^{-}
$$

which is clearly equivalent to Eq. (56). The eigenvalues of the BCS Hamiltonian, Eq. (63), are given by Eq. (59) where the parameters $E_{m}$ are the solutions of the Richardson's equations, Eq. (58), with $\Omega_{\mathbf{k}}=2$. One needs to take into account the fact that for each $\mathbf{k}$ there is $\mathbf{a}-\mathbf{k}$ in those sums. Moreover, if the crystal has space-inversion symmetry $\varepsilon_{\mathbf{k}}=\varepsilon_{-\mathbf{k}}$. This additional symmetry, which converts each single-particle level into a four-fold degenerate one, may have dramatic consequences. For example, the numerical solution for the ground state in the BCS case is free of singularities due to the fact that the pair energies $E_{m}$ come in complex conjugate pairs for any value of the coupling strength $g$.

In previous work [12], a gauge $S U(2)$ symmetry was identified. The $S U(2)$ symmetry generators are the local operators

$$
S_{\mathbf{k}}^{+}=c_{\mathbf{k} \uparrow}^{\dagger} c_{-\mathbf{k} \downarrow}=\left(S_{\mathbf{k}}^{-}\right)^{\dagger}, \quad S_{\mathbf{k}}^{z}=\frac{1}{2}\left(n_{\mathbf{k} \uparrow}-n_{-\mathbf{k} \downarrow}\right),
$$




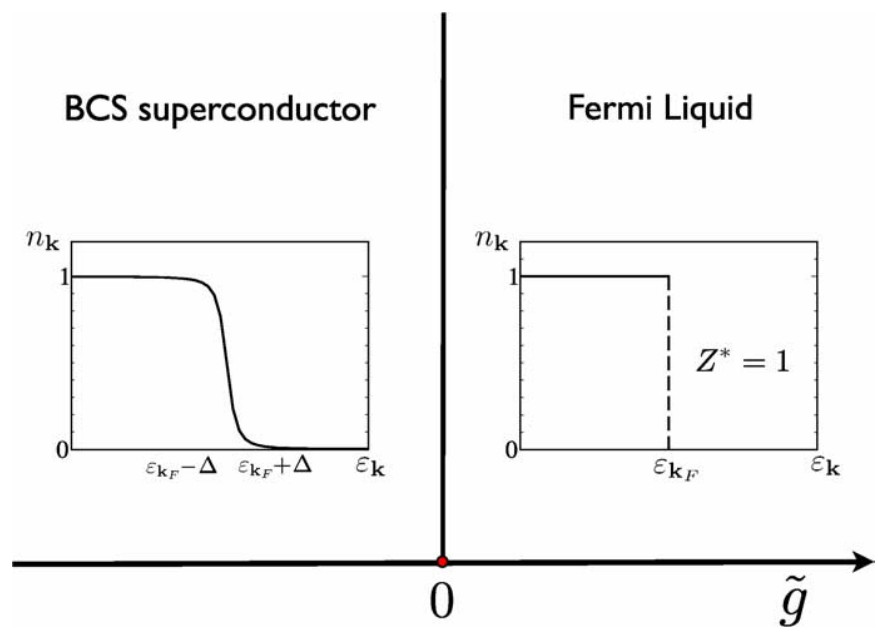

Fig. 1. Quantum phase diagram of the BCS model as a function of the interaction strength $\tilde{g}$ in the thermodynamic, $N \rightarrow \infty$, limit. The insets represent the single-particle occupation number $n_{\mathbf{k}}$ in each quantum phase. Notice that, for positive $\tilde{g}$, the Fermi liquid quasiparticle renormalization factor $Z^{*}$ is unity regardless of the magnitude of the interaction.

which commute with the pseudospins $\tau_{\mathbf{k}}$, i.e., $\left[S_{\mathbf{k}}^{\mu}, \tau_{\mathbf{k}^{\prime}}^{\nu}\right]=0$, for $\mu, v= \pm, z$. This symmetry amounts to the conservation of the charge parity per mode pair ( $\mathbf{k} \uparrow,-\mathbf{k} \downarrow)$. Indeed, this local symmetry is responsible for the Pauli blocking of the (unpair) singlyoccupied states. We would like to emphasize that all the symmetry analysis applied to Eq. (63) is also applicable, after proper rewriting of the symmetry operators, to Eq. (56).

It is interesting to analyze the quantum phase diagram of the BCS Hamiltonian $H_{\mathrm{BCS}}$ as a function of the coupling strength $\tilde{g}=g N$. To this end one needs to study the behavior of the quantum correlations of the ground state in the thermodynamic limit. It has been shown, under quite general assumptions, that the Bethe equations of the integrable BCS Hamiltonian in the thermodynamic limit are the BCS equations [33,34]. The condensation energy for attractive pairing in this limit is $E_{\text {cond }}=-\frac{2 \omega_{D}}{d} e^{2 / \tilde{g}}$ where $d$ is the mean level spacing $\left(\sim \mathrm{Vol}^{-1}\right)$ and $\omega_{D}$ is the Debye frequency cutoff. For repulsive pairing $E_{\text {cond }}=0$. It turns out that there is a quantum phase transition between a BCS superconductor (broken $U$ (1) symmetry) and a Fermi liquid of a peculiar type at $\tilde{g}=0$ (see Fig. 1). It is important to emphasize that the ground state energy has an essential singularity at $\tilde{g}=0^{-}$, implying that it is a continuous infinite-order (Kosterlitz-Thouless-like [17]) quantum phase transition but with a broken $U(1)$ symmetry. (Notice that this result is independent of the spacedimensionality of the problem.) We have numerically solved the Bethe equations and found that the Fermi liquid has quasiparticle renormalization factor $Z^{*}=1$ independently of the magnitude of $\tilde{g}$; moreover, it displays enhanced superconducting fluctuations but it is not a superconductor. The fact that $Z^{*}=1$ has been previously remarked in [35] using functional integrals. 
The exactly solvable Hamiltonian Eq. (63) can be generalized to the case of multibands in the following way

$$
H_{\mathrm{BCSn}}=\sum_{\mathfrak{n} \mathbf{k}} \varepsilon_{\mathbf{k}}^{\mathfrak{n}}\left(n_{\mathfrak{n} \mathbf{k} \uparrow}+n_{\mathfrak{n}-\mathbf{k} \downarrow}\right)+\sum_{\mathfrak{n} \mathbf{k} \mathfrak{n}^{\prime} \mathbf{k}^{\prime}}^{\prime} g_{\mathfrak{n} \mathfrak{n}^{\prime}} c_{\mathfrak{n} \mathbf{k} \uparrow}^{\dagger} c_{\mathfrak{n}-\mathbf{k} \downarrow}^{\dagger} c_{\mathfrak{n}^{\prime}-\mathbf{k}^{\prime} \downarrow} c_{\mathfrak{n}^{\prime} \mathbf{k}^{\prime} \uparrow},
$$

where $\mathfrak{n}$ represents the band index, $n_{\mathfrak{n} \mathbf{k} \sigma}=c_{\mathfrak{n} \mathbf{k} \sigma}^{\dagger} c_{\mathfrak{n} \mathbf{k} \sigma}$, and $g_{\mathfrak{n} \mathfrak{n}^{\prime}}=g_{\mathfrak{n}^{\prime} \mathfrak{n}}$. The prime in the sum means that the terms $(\mathfrak{n}, \mathbf{k})=\left(\mathfrak{n}^{\prime}, \mathbf{k}^{\prime}\right)$ are excluded. Global symmetries of the model include $\tau^{z}=\sum_{\mathfrak{n}, \mathbf{k}} \tau_{\mathfrak{n} \mathbf{k}}^{z}$ and $\sum_{\mathfrak{n}, \mathbf{k}}\left(\tau_{\mathfrak{n} \mathbf{k}}^{z}\right)^{2}$ with $\tau_{\mathfrak{n} \mathbf{k}}^{z}=\frac{1}{2}\left(n_{\mathfrak{n} \mathbf{k} \uparrow}+n_{\mathfrak{n}-\mathbf{k} \downarrow}-1\right)$. The local $S U(2)$ symmetry has as generators

$$
S_{\mathfrak{n} \mathbf{k}}^{+}=c_{\mathfrak{n} \mathbf{k} \uparrow}^{\dagger} c_{\mathfrak{n}-\mathbf{k} \downarrow}=\left(S_{\mathfrak{n} \mathbf{k}}^{-}\right)^{\dagger}, \quad S_{\mathfrak{n} \mathbf{k}}^{z}=\frac{1}{2}\left(n_{\mathfrak{n} \mathbf{k} \uparrow}-n_{\mathfrak{n}-\mathbf{k} \downarrow}\right) .
$$

Clearly, the BCS Hamiltonian of Eq. (63) is a particular case of Eq. (68) for a single band $(\mathfrak{n}=1)$. It is straightforward to see that $H_{\mathrm{BCS} \mathfrak{n}}$ is exactly solvable for interactions:

(1) $g_{\mathfrak{n} \mathfrak{n}^{\prime}}=\delta_{\mathfrak{n} \mathfrak{n}^{\prime}} g_{\mathfrak{n}}$ (decoupled BCS bands);

(2) $g_{\mathfrak{n} \mathfrak{n}^{\prime}}=g$ (effective one-band BCS model).

For the particular case of two bands, the Hamiltonian equation (68) might be of interest to describe the phenomenon of two-gap superconductivity recently observed in materials like $\mathrm{MgB}_{2}$. We may consider here an $S U(2)_{1} \otimes S U(2)_{2}$ structure, with each $S U(2)_{\mathfrak{n}}$ generated by the elements

$$
\tau_{\mathfrak{n}}^{+}=\sum_{\mathbf{k}} c_{\mathfrak{n} \mathbf{k} \uparrow}^{\dagger} c_{\mathfrak{n}-\mathbf{k} \downarrow}^{\dagger}=\left(\tau_{\mathfrak{n}}^{-}\right)^{\dagger}, \quad \tau_{\mathfrak{n}}^{z}=\sum_{\mathbf{k}} \tau_{\mathfrak{n} \mathbf{k}}^{z} .
$$

The case of two flat bands $\varepsilon=-\varepsilon_{\mathbf{k}}^{1}=\varepsilon_{\mathbf{k}}^{2}$ with equal diagonal interaction terms, i.e., $g_{11}=$ $g_{22}$, can be easily shown to be exactly solvable: by using the quantum invariants of the $X X Z$ Richardson-Gaudin (RG) models

$$
R_{\mathfrak{n}}=\tau_{\mathfrak{n}}^{z}+2 \sum_{\mathfrak{n}^{\prime} \neq \mathfrak{n}}\left[\frac{X_{\mathfrak{n} \mathfrak{n}^{\prime}}}{2}\left(\tau_{\mathfrak{n}}^{+} \tau_{\mathfrak{n}^{\prime}}^{-}+\tau_{\mathfrak{n}}^{-} \tau_{\mathfrak{n}^{\prime}}^{+}\right)+Z_{\mathfrak{n} \mathfrak{n}^{\prime}} \tau_{\mathfrak{n}}^{z} \tau_{\mathfrak{n}^{\prime}}^{z}\right]
$$

it can be shown that the two-band pairing Hamiltonian is equivalent (up to an overall constant) to

$$
H_{\mathrm{BCS} 2}=2 \varepsilon\left(\tau_{2}^{z}-\tau_{1}^{z}\right)+g_{11}\left(\tau_{1}^{+} \tau_{1}^{-}+\tau_{2}^{+} \tau_{2}^{-}\right)+g_{12}\left(\tau_{2}^{+} \tau_{1}^{-}+\tau_{1}^{+} \tau_{2}^{-}\right),
$$

where $g_{11}=4 \varepsilon Z_{21}$ and $g_{12}=4 \varepsilon X_{21}$ are two arbitrary real numbers (with the parametrization of Eq. (14), $\left.g_{12}^{2}-g_{11}^{2}=(4 \varepsilon g)^{2} s\right)$. To arrive to expression (71) we have used the Casimir invariants

$$
\frac{1}{2}\left(\tau_{\mathfrak{n}}^{+} \tau_{\mathfrak{n}}^{-}+\tau_{\mathfrak{n}}^{-} \tau_{\mathfrak{n}}^{+}\right)+\left(\tau_{\mathfrak{n}}^{z}\right)^{2}=\mathrm{S}(\mathrm{S}+1)
$$

together with the conservation of $\tau^{z}=\tau_{1}^{z}+\tau_{2}^{z}$ and $\left(\tau_{1}^{z}\right)^{2}+\left(\tau_{2}^{z}\right)^{2}$. This is the Hamiltonian originally proposed by Suhl, Matthias and Walker [6] as an extension of the BCS model, to include situations where the scattering between electrons from different bands contributes substantially to the resistivity in the normal state. 
Table 2

Single-particle energies and degeneracies for the Tin isotopes in the $N=50-82$ shell

\begin{tabular}{llllll}
\hline s. p. level & $\mathrm{d}_{5 / 2}$ & $\mathrm{~g}_{7 / 2}$ & $\mathrm{~s}_{1 / 2}$ & $\mathrm{~d}_{3 / 2}$ & $\mathrm{~h}_{11 / 2}$ \\
\hline s. p. energy (MeV) & 0.0 & 0.22 & 1.90 & 2.20 & 2.80 \\
s. p. degeneracy & 6 & 8 & 2 & 4 & 12 \\
\hline
\end{tabular}

Table 3

Single-particle energies and degeneracies for electrons in a $6 \times 6$ square lattice

\begin{tabular}{llllllllll}
\hline s. p. energy & -4 & -3 & -2 & -1 & 0 & 1 & 2 & 3 & 4 \\
s. p. degeneracy & 2 & 8 & 8 & 8 & 20 & 8 & 8 & 8 & 2 \\
\hline
\end{tabular}

The (unnormalized) eigenstates of $H_{\mathrm{BCS} 2}$ are given by (with $t_{1}=-\eta$ and $t_{2}=\eta$ )

$$
|\Psi\rangle=\prod_{\ell=1}^{M}\left(\frac{1}{E_{\ell}+\eta} \tau_{1}^{+}+\frac{1}{E_{\ell}-\eta} \tau_{2}^{+}\right)|v\rangle
$$

where the spectral parameters $E_{\ell}$ satisfy Bethe's equations $\left(d_{ \pm}=d_{1} \pm d_{2}\right.$, and $2 d_{1(2)}=$ $\left.v_{1(2)}-\Omega_{1(2)} / 2\right)$

$$
\varepsilon+\eta \frac{g_{12} d_{+} E_{\ell}-2 \varepsilon g d_{-}\left(1+s E_{\ell}^{2}\right)}{E_{\ell}^{2}-\varepsilon^{2}}+2 g \varepsilon \sum_{n(\neq \ell)=1}^{M} \frac{1+s E_{\ell} E_{n}}{E_{\ell}-E_{n}}=0 .
$$

The corresponding eigenvalues can be easily obtained from those of the integrals of motion.

More complex situations arise in the application of the BCS model to the spherical nuclear shell model or to finite lattices. As an example in nuclear physics we will consider the semi-magic Sn isotopes. These series of nuclei can be modelled by a set of valence neutrons occupying the single-particle orbits in the $N=50-82$ shell interacting with a residual BCS Hamiltonian. In Table 2 we show the experimental single-particle energies and the corresponding degeneracies in the spherical single-particle basis.

Richardson's equations for this case have non-equally spaced levels and variable degeneracies. While the solution to this problem has been found using standard techniques [36], larger systems would require more sophisticated methods [32] to avoid the singularities.

A quite similar situation arises in solving the BCS Hamiltonian in finite twodimensional lattices of size $L \times L$ [9]. The single particle energies in units of the hopping matrix element are $\varepsilon_{k}=-2\left(\cos k_{x}+\cos k_{y}\right)$, with $k_{\rho}=2 \pi n_{\rho} / L$ and $-L / 2 \leqslant n_{\rho}<L / 2$. Table 3 shows the single particle energies and degeneracies for a $6 \times 6$ lattice.

Numerical applications of the RG models to fermionic problems in nuclear physics and condensed matter have been concentrated on the BCS Hamiltonian with uniform couplings. The use of the hyperbolic model, a particular solution of the $X X Z$ generalized Gaudin models, with non-uniform coupling strength has been suggested in Ref. [37] to describe the physics of multigrain systems, though no practical applications have been carried out so far.

Another exactly-solvable model with a separable pairing interaction (SPI) was proposed by Pan, Draayer and Ormand [38]. The Hamiltonian has degenerate single-particle energies 
but some structure in the pairing interaction

$$
H_{\mathrm{SPI}}=\varepsilon \sum_{\mathbf{j}} n_{\mathbf{j}}+\sum_{\mathbf{j}, \mathbf{j}^{\prime}, m, m^{\prime}} g_{\mathbf{j} \mathbf{j}^{\prime}} a_{\mathbf{j} m}^{\dagger} a \frac{\dagger}{\mathbf{j}^{m} m} a_{\overline{\mathbf{j}^{\prime} m^{\prime}}} a_{\mathbf{j}^{\prime} m^{\prime}},
$$

with $g_{\mathbf{j} \mathbf{j}^{\prime}}=g c_{\mathbf{j}} c_{\mathbf{j}}^{\prime}$. This model can be derived from the model of Eq. (46) using the parametrization of Eq. (22) and taking $\varepsilon_{\mathbf{j}}=c_{\mathbf{j}}^{2}$. Inserting the number operator divided by the number of particles and adjusting the interaction strength, one can cancel out the one-body and two-body diagonal parts in the Hamiltonian.

Apart from its relevance in the nuclear shell model, the SPI model has also been used in connection with atomic BECs [39] and for establishing variational lower bounds on the energy of general two-body Hamiltonians [40].

\subsection{Particle-hole-like models}

It is clear that what is behind the exact solvability of these different models is a GGA and the existence of certain quantum invariants. Different representations of the Gaudin operators lead to different models but all of them with the same dynamics. In this section, we continue with $s u(2)$ fermionic representation models.

In previous section we have written down BCS-like models using an $s u(2)$ representation in terms of pseudospins $\tau$. We have also seen that there is another fermionic representation for $s u(2)$ in terms of the generators of Eq. (67). The natural question that arises is: can we write down sensible exactly solvable models of interacting fermions in terms of this $s u(2)$ representation? and the simple answer is yes.

The Lipkin-Meshkov-Glick (LMG) model [7] was originally introduced to study phase transitions in finite nuclei. The model considers $N$ fermions distributed in two $N$-fold degenerate levels (termed upper and lower shells). The latter are separated by an energy gap $\varepsilon$

$$
\begin{aligned}
H_{\mathrm{LMG}}= & \frac{\varepsilon}{2} \sum_{\mathbf{k} \sigma} \sigma c_{\mathbf{k} \sigma}^{\dagger} c_{\mathbf{k} \sigma}+\frac{V}{2 N} \sum_{\mathbf{k k}^{\prime} \sigma} c_{\mathbf{k} \sigma}^{\dagger} c_{\mathbf{k}^{\prime} \sigma}^{\dagger} c_{\mathbf{k}^{\prime}-\sigma} c_{\mathbf{k}-\sigma} \\
& +\frac{W}{2 N} \sum_{\mathbf{k k}^{\prime} \sigma} c_{\mathbf{k} \sigma}^{\dagger} c_{\mathbf{k}^{\prime}-\sigma}^{\dagger} c_{\mathbf{k}^{\prime} \sigma} c_{\mathbf{k}-\sigma},
\end{aligned}
$$

with the quantum number $\sigma= \pm$ labelling the level. In Eq. (76) the interaction term $V$ scatters a pair of particles across the Fermi level, i.e., it is a two particle-hole interaction, while the term $W$ exchange particles in the two levels. Upon introducing the collective particle-hole operators

$$
S^{+}=\sum_{\mathbf{k}} c_{\mathbf{k}+}^{\dagger} c_{\mathbf{k}-}=\left(S^{-}\right)^{\dagger}, \quad S^{z}=\frac{1}{2} \sum_{\mathbf{k} \sigma} \sigma c_{\mathbf{k} \sigma}^{\dagger} c_{\mathbf{k} \sigma},
$$

which satisfy the $s u(2)$ commutation relations, Eq. (76) may be rewritten as

$$
H_{\mathrm{LMG}}=\varepsilon S^{z}+\frac{V}{2 N}\left(S^{+} S^{+}+S^{-} S^{-}\right)+\frac{W}{2 N}\left(S^{+} S^{-}+S^{-} S^{+}\right) .
$$


As defined by Eq. (78), $H_{\mathrm{LMG}}$ is invariant under the inversion symmetry operation $I$ that transforms $\left(S^{x}, S^{y}, S^{z}\right) \mapsto\left(-S^{x},-S^{y}, S^{z}\right)$, and it also commutes with the (Casimir) operator $S^{2}=\left(S^{+} S^{-}+S^{-} S^{+}\right) / 2+\left(S^{z}\right)^{2}$. Thus, Eq. (78) is equivalent to

$$
H_{\mathrm{LMG}}=\varepsilon S^{z}+\frac{V}{2 N}\left(S^{+} S^{+}+S^{-} S^{-}\right)-\frac{W}{N} S^{z} S^{z}+\frac{W}{N} S^{2} .
$$

The Hamiltonian $H_{\mathrm{LMG}}$ has a band matrix representation in an $s u(2)$ basis, and it can be easily diagonalized for large values of $N$. As such, the model has been used as a testing ground for many-body approximations in nuclear physics. More recently, the simplicity of the model and the fact that it can be interpreted as a Heisenberg chain with long range exchange interactions, made it fashionable to study relations between entanglement and quantum phase transitions.

We will show in Section 6.3.2 that the LMG model is exactly solvable. But before consider the modified problem

$$
H_{\mathrm{p}-\mathrm{h}}=\sum_{\mathbf{k} \sigma} \frac{\varepsilon_{\mathbf{k}}}{2} \sigma c_{\mathbf{k} \sigma}^{\dagger} c_{\mathbf{k} \sigma}+\frac{W}{2 N} \sum_{\mathbf{k k}^{\prime} \sigma} c_{\mathbf{k} \sigma}^{\dagger} c_{\mathbf{k}^{\prime}-\sigma}^{\dagger} c_{\mathbf{k}^{\prime} \sigma} c_{\mathbf{k}-\sigma},
$$

where $\sigma= \pm$ may be now interpreted as a band index. Let us introduce the following commuting $s u(2)$ algebras

$$
\begin{array}{ll}
S_{\mathbf{k}}^{+}=c_{\mathbf{k}+}^{\dagger} c_{\mathbf{k}-}=\left(S_{\mathbf{k}}^{-}\right)^{\dagger}, & S_{\mathbf{k}}^{z}=\frac{1}{2}\left(n_{\mathbf{k}+}-n_{\mathbf{k}-}\right), \\
\tau_{\mathbf{k}}^{+}=c_{\mathbf{k}+}^{\dagger} c_{\mathbf{k}-}^{\dagger}=\left(\tau_{\mathbf{k}}^{-}\right)^{\dagger}, & \tau_{\mathbf{k}}^{z}=\frac{1}{2}\left(n_{\mathbf{k}+}+n_{\mathbf{k}-}-1\right),
\end{array}
$$

in terms of which $H_{\mathrm{p}-\mathrm{h}}$ can be written (up to an irrelevant constant) as

$$
H_{\mathrm{p}-\mathrm{h}}=\sum_{\mathbf{k}} \varepsilon_{\mathbf{k}} S_{\mathbf{k}}^{z}+\frac{W}{N} \sum_{\mathbf{k k}^{\prime}} S_{\mathbf{k}}^{+} S_{\mathbf{k}^{\prime}}^{-}
$$

$H_{\mathrm{p}-\mathrm{h}}$ is dynamically equivalent to $H_{\mathrm{BCS}}$ and, thus, it is also exactly solvable.

\section{6. $\bigoplus_{\mathrm{l}} \operatorname{su}(1,1)$ bosonic representation models}

\subsection{Bosonic BCS-like models}

The boson BCS or pairing Hamiltonian can be written in complete analogy to the fermion case, Eq. (53), as

$$
H_{\mathrm{BBCS}}=\sum_{\mathbf{l}} \varepsilon_{\mathbf{l}} n_{\mathbf{l}}+\frac{g}{4} \sum_{\mathbf{I I}^{\prime}} b_{\mathbf{1}}^{\dagger} b_{\overline{\mathbf{1}}}^{\dagger} b_{\overline{\mathbf{l}}^{\prime}} b_{\mathbf{I}^{\prime}},
$$

where $b_{\mathbf{1}}^{\dagger}\left(b_{\mathbf{I}^{\prime}}\right)$ creates (destroys) a boson in the state $\mathbf{l}$, and $n_{\mathbf{l}}=b_{\mathbf{1}}^{\dagger} b_{\mathbf{l}}$ is the number operator. For simplicity, we will consider here scalar bosons, but an arbitrary internal spin can be easily taken into account as in the case of fermions. The label $\mathbf{l}$ is a short-hand notation for a set of quantum numbers; for example, the states of a 3D isotropic harmonic oscillator 
potential are labelled by $\mathbf{l} \equiv(n l m)$, where $n$ is the oscillator quantum number, $l$ is the orbital angular momentum and $m$ its third projection. In Eq. (84) $\overline{\mathbf{I}}$ refers to the timereversed state of $\mathbf{l}$. Following (54) the time-reversed annihilation boson operator is $b_{\overline{\mathbf{l}}}=$ $b \overline{n l m}=(-1)^{l-m} b_{n l-m}$.

In the following we will be concerned with spin scalar bosons, a possibility that cannot be realized in fermionic models. It is worth emphasizing, however, that the exact solution for boson systems can easily incorporate the spin degree of freedom (integer spin), and there might be important applications for spinor BECs [41] not explored so far.

Once again, Richardson [42] determined the complete spectrum of the boson BCS Hamiltonian of Eq. (84). This work also escaped the attention of the physics community until very recently, when the model was shown to be quantum integrable [9]. Exactlysolvable generalizations of the uniform pairing Hamiltonian were proposed and subsequently applied to various finite Bose systems $[43,44]$. In analogy with the fermionic systems presentation of previous sections, we will first introduce a specific representation of the $\operatorname{su}(1,1)$ generators

$$
K_{\mathbf{1}}^{+}=\frac{1}{2} b_{\mathbf{1}}^{\dagger} b_{\mathbf{1}}^{\dagger}=\left(K_{\mathbf{1}}^{-}\right)^{\dagger}, \quad K_{\mathbf{l}}^{z}=\frac{1}{2} b_{\mathbf{1}}^{\dagger} b_{\mathbf{l}}+\frac{1}{4} \Omega_{\mathbf{l}},
$$

where the operator $K_{\mathbf{I}}^{+}$creates a pair of fermions in time-reversal states and $\Omega_{\mathbf{l}}=2 K_{\mathbf{l}}+1$ is the degeneracy of the state $\mathbf{l}$ related to the pseudospin $K_{\mathbf{l}}$. The operators of Eq. (85) satisfy the $s u(1,1)$ commutation relations

The BCS Hamiltonian $H_{\mathrm{BBCS}}$ can be derived from the rational family (17) as a linear combination of the integrals of motion

$$
H_{\mathrm{BBCS}}=\sum_{\mathbf{I}} \varepsilon_{\mathbf{l}} R_{\mathbf{l}}(\varepsilon)+C=\sum_{\mathbf{I}} \varepsilon_{\mathbf{l}}\left(2 K_{\mathbf{l}}^{z}-\frac{1}{2} \Omega_{\mathbf{l}}\right)+g \sum_{\mathbf{I I}^{\prime}} K_{\mathbf{l}}^{+} K_{\mathbf{I}^{\prime}}^{-} .
$$

The complete set of eigenstates of this model are given by the product wavefunction

$$
|\Psi\rangle=\prod_{m=1}^{M} \mathrm{~S}_{m}^{+}|\nu\rangle, \quad \mathrm{S}_{m}^{+}=\sum_{\mathbf{l}} X_{m \mathbf{l}} K_{\mathbf{l}}^{+}=\sum_{\mathbf{l}} \frac{1}{2 \varepsilon_{\mathbf{l}}-E_{m}} K_{\mathbf{l}}^{+},
$$

where $|v\rangle \equiv\left|v_{1}, v_{2}, \ldots, v_{L}\right\rangle$, with $L$ the total number of single particle states, is a state of $v$ unpaired bosons $\left(v=\sum_{\mathbf{l}} v_{\mathbf{l}}\right)$ defined by

$$
K_{\mathbf{l}}^{-}|v\rangle=0, \quad n_{\mathbf{l}}|v\rangle=v_{\mathbf{l}}|v\rangle
$$

$\nu_{\mathbf{l}}$ are referred to the seniority quantum numbers.

The total number of particles is $N=2 M+v$, with $M$ the number of paired bosons. Each eigenstate $|\Psi\rangle$ is completely defined by a set of $M$ spectral parameters (pair energies) $E_{m}$ which are a particular solution of the Richardson's equations

$$
1+\frac{g}{2} \sum_{\mathbf{l}=1}^{L} \frac{\Omega_{\mathbf{l}}+2 \nu_{\mathbf{l}}}{2 \varepsilon_{\mathbf{l}}-E_{m}}-2 g \sum_{\ell(\neq m)=1}^{M} \frac{1}{E_{m}-E_{\ell}}=0,
$$


and their eigenvalues are given by

$$
E=\sum_{\mathbf{l}=1}^{L} \varepsilon_{\mathbf{l}} v_{\mathbf{l}}+\sum_{m=1}^{M} E_{m}
$$

Hamiltonians constructed as general linear combinations of the integrals of motion $H=\sum_{\mathbf{l}=1} \varepsilon_{\mathbf{l}} R_{\mathbf{l}}(\eta)$ have eigenvalues $E=\sum_{\mathbf{l}=1}^{L} \varepsilon_{\mathbf{l}}\left(r_{\mathbf{l}}+v_{\mathbf{l}}\right)$ where $r_{\mathbf{l}}$ is the eigenvalue of the integral of motion $R_{\mathbf{I}}$ [44].

\subsection{Pairing Hamiltonians for bosons in confining traps}

As an application of the boson rational family, we will consider the problem of a boson system confined to a harmonic-oscillator trap and subject to boson pairing interactions [43]. The pairing Hamiltonian with uniform couplings cannot describe the physics of a trapped boson system, for the following reason. Looking back at the commutators of the pair operators, Eq. (38), we see that they are proportional to the degeneracy of the state $\mathbf{l}$ that appears inside the definition of the generator $K_{1}^{z}$ (Eq. (85)). Thus, the matrix elements of the pairing Hamiltonian between states $\mathbf{l}$ and $\mathbf{l}^{\prime}$ will be proportional to $\sqrt{\Omega_{\mathbf{l}} \Omega_{\mathbf{l}^{\prime}}}$. In a $3 \mathrm{D}$ harmonic oscillator with $\mathbf{l} \equiv(\mathrm{nlm})$, the shell degeneracy is $\Omega_{\mathbf{l}} \sim n^{2}$. On the other hand, the single-particle energies are $\varepsilon_{\mathbf{l}}=n$. Thus, the net effect would be the scattering of boson pairs to high-lying levels with greater probability than to low-lying levels, producing unphysical occupation numbers. This was precisely the behavior observed in a numerical solution of Richardson's equations (88) for a system of 1000 bosons with an attractive pairing strength $g$ [43].

We can use the freedom we have in choosing the parameters $\eta_{\mathbf{l}}$ entering in the definition of the $R_{\mathbf{I}}$ operators to obtain a physically relevant exactly-solvable model. In order to cancel out the unphysical dependence of the pair-coupling matrix elements on the degeneracies, we choose the $\eta_{\mathbf{l}}$ 's so that $\eta_{\mathbf{I}}=\left(\varepsilon_{\mathbf{l}}\right)^{3}$. The Hamiltonian, which is given by the linear combination of the new $R_{\mathbf{l}}$ 's is

$$
H_{\mathrm{TB}}=2 \sum_{\mathbf{I}} \varepsilon_{\mathbf{l}} R_{\mathbf{I}}=C+\sum_{\mathbf{I}} \bar{\varepsilon}_{\mathbf{l}} n_{\mathbf{l}}+\sum_{\mathbf{l} \neq \mathbf{I}^{\prime}} g_{\mathbf{I I}^{\prime}}\left[K_{\mathbf{l}}^{+} K_{\mathbf{I}^{\prime}}^{-}-n_{\mathbf{l}} n_{\mathbf{I}^{\prime}}\right],
$$

where

$$
\begin{aligned}
& C=\frac{1}{2} \sum_{\mathbf{I}} \varepsilon_{\mathbf{l}} \Omega_{\mathbf{l}}-\frac{1}{4} \sum_{\mathbf{l} \neq \mathbf{I}^{\prime}} g_{\mathbf{I I}^{\prime}} \Omega_{\mathbf{l}} \Omega_{\mathbf{I}^{\prime}}, \quad \bar{\varepsilon}_{\mathbf{I}}=\varepsilon_{\mathbf{l}}-\sum_{\mathbf{I}^{\prime}(\neq \mathbf{l})} g_{\mathbf{I}^{\prime}} \Omega_{\mathbf{I}^{\prime}}, \\
& g_{\mathbf{I I}^{\prime}}=\frac{g}{2} \frac{1}{\varepsilon_{\mathbf{l}}^{2}+\varepsilon_{\mathbf{I}^{\prime}}^{2}+\varepsilon_{\mathbf{I}} \varepsilon_{\mathbf{I}^{\prime}}} .
\end{aligned}
$$

The interaction in Eq. (91) has the nice feature that its two-body matrix elements decrease with the number of shells, as one would expect in general. It has the particular property that the interactions of the pair- and density-fluctuations are strictly the same but opposite in sign. Taking into account that $\varepsilon_{\mathbf{l}}$ is proportional to $n$, the two-body matrix elements in Eq. (90) cancel out the dependence on the degeneracies in the effective pair-coupling matrix elements. Thus, $H_{\mathrm{TB}}$ should be more appropriate than $H_{\mathrm{BBC}}$ when modelling a harmonically-confined boson system with a pairing-like interaction. 
The spectrum of $H_{\mathrm{TB}}$ can be obtained from the eigenvalues $r_{\mathbf{I}}$ of the associated $R_{\mathbf{I}}$ operators as $E=2 \sum_{\mathbf{l}} \varepsilon_{\mathbf{l}} r_{\mathbf{l}}$, with the end result being

$$
E=\frac{1}{2} \sum_{\mathbf{l}} \varepsilon_{\mathbf{l}} \Omega_{\mathbf{l}}-\frac{1}{4} \sum_{\mathbf{l} \neq \mathbf{I}^{\prime}} g_{\mathbf{I}^{\prime}} \Omega_{\mathbf{l}} \Omega_{\mathbf{I}^{\prime}}-2 g \sum_{\mathbf{l} p} \frac{\varepsilon_{\mathbf{l}} \Omega_{\mathbf{l}}}{2 \varepsilon_{\mathbf{l}}^{3}-E_{p}} .
$$

(Note that the first two terms of Eq. (92) exactly cancel the constant term $C$ in Eq. (90).)

We solved Richardson's equations for $H_{\mathrm{TB}}$ for a system of $M=500$ boson pairs and $L=50$ oscillator shells. In this case, the occupation numbers display a reasonable physical pattern, with the occupancies decreasing monotonically with increasing single-boson energy [43].

In the case of repulsive pairing a highly unexpected feature was found [43]. For small values of $g$ the system behaves as a normal BEC. At a critical value of the pairing strength $g_{c}$ a second-order quantum phase transition takes place. The new phase is characterized by a fragmentation of the condensate with the two lowest states macroscopically occupied, while the occupation of the other levels is negligible.

\subsection{Exactly-solvable two-level boson Hamiltonians}

The restriction of the bosonic RG models to two-levels comprises several well-known quantum models. Among them we will discuss the interacting boson model [13], the LMG model [7] and the two Josephson-coupled BECs Hamiltonian [14]. Let us begin by defining the two integrals of motion from the most general $X X Z \mathrm{RG}$ models

$$
\begin{aligned}
& R_{a}=K_{a}^{z}-X_{12}\left[K_{a}^{+} K_{b}^{-}+K_{a}^{-} K_{b}^{+}\right]+2 Z_{12} K_{a}^{z} K_{b}^{z}, \\
& R_{b}=K_{b}^{z}+X_{12}\left[K_{a}^{+} K_{b}^{-}+K_{a}^{-} K_{b}^{+}\right]-2 Z_{12} K_{a}^{z} K_{b}^{z},
\end{aligned}
$$

where the operators $K_{1}^{\kappa}$ are defined in Eqs. (85). From Eqs. (93) we observe that the sum gives the total number of bosons which is a conserved quantity. We are then left with one independent quantum invariant, that we can take as the difference between the two integrals of motion to define the Hamiltonian

$$
\begin{aligned}
H_{\mathrm{B} 2} & =\varepsilon\left(R_{b}-R_{a}\right) \\
& =\varepsilon\left(K_{b}^{z}-K_{a}^{z}\right)+2 \varepsilon\left[X_{12}\left(K_{a}^{+} K_{b}^{-}+K_{a}^{-} K_{b}^{+}\right)-2 Z_{12} K_{a}^{z} K_{b}^{z}\right] .
\end{aligned}
$$

Using Eq. (85) we rewrite $H_{\mathrm{B} 2}$ as

$$
\begin{aligned}
H_{\mathrm{B} 2}= & \frac{\varepsilon}{2}\left[\left(1-Z_{12} \Omega_{a}\right) n_{b}-\left(1+Z_{12} \Omega_{b}\right) n_{a}\right]+v \sum_{\alpha, \beta}\left(b_{\beta}^{\dagger} b_{\bar{\beta}}^{\dagger} a_{\bar{\alpha}} a_{\alpha}+a_{\alpha}^{\dagger} a_{\bar{\alpha}}^{\dagger} b_{\bar{\beta}} b_{\beta}\right) \\
& +w n_{b} n_{a}+C,
\end{aligned}
$$

where $v=\frac{\varepsilon}{2} X_{12}$ and $w=-\varepsilon Z_{12}$ are two arbitrary real numbers, and $b_{\beta}^{\dagger}\left(a_{\alpha}^{\dagger}\right)$ creates a boson in level $b(a)$ with an internal quantum number $\beta(\alpha)$. As usual the bar in the internal labels means a time-reversed state, and $\Omega_{b(a)}$ is the degeneracy of the level. The constant term is $C=\frac{\varepsilon}{4}\left(\Omega_{b}-\Omega_{a}-Z_{12} \Omega_{b} \Omega_{a}\right)$. Using the parametrization of Eq. (14) (with $t_{1}=-\eta$ and $\left.t_{2}=\eta\right), 4 v^{2}-w^{2}=s g^{2} \varepsilon^{2}$, which can be positive, negative or zero depending upon the choice of parameters. 
The (unnormalized) eigenstates of $H_{\mathrm{B} 2}$ are given by

$$
|\Psi\rangle=\prod_{\ell=1}^{M}\left(\frac{1}{E_{\ell}+\eta} K_{a}^{+}+\frac{1}{E_{\ell}-\eta} K_{b}^{+}\right)|v\rangle,
$$

with spectral parameters satisfying Bethe's equations

$$
\varepsilon-2 \eta \frac{4 v d_{+} E_{\ell}+\varepsilon g d_{-}\left(1+s E_{\ell}^{2}\right)}{E_{\ell}^{2}-\varepsilon^{2}}+2 g \varepsilon \sum_{n(\neq \ell)=1}^{M} \frac{1+s E_{\ell} E_{n}}{E_{\ell}-E_{n}}=0,
$$

where $d_{ \pm}=d_{a} \pm d_{b}$, and $2 d_{a(b)}=v_{a(b)}+\Omega_{a(b)} / 2$. The corresponding eigenvalues can be constructed from the integrals of motion eigenvalues as

$$
E_{\mathrm{B} 2}=4 d_{a} d_{b} w-\varepsilon d_{-}-2 \eta \sum_{\ell=1}^{M} \frac{4 v d_{-} E_{\ell}+\varepsilon g d_{+}\left(1+s E_{\ell}^{2}\right)}{E_{\ell}^{2}-\varepsilon^{2}} .
$$

We will discuss next the application of the two-level RG bosonic models to three wellknown quantum models.

\subsubsection{The interacting boson model}

The interacting boson model (IBM) has been a highly successful phenomenological model to describe the collective properties of medium and heavy nuclei. The IBM captures the collective dynamics of nuclear systems by representing correlated pairs of nucleons with angular momentum $\hat{L}$ by ideal bosons with the same angular momentum. In its simplest version, known as IBM1, there is no distinction between protons and neutrons and only angular momentum $\hat{L}=0(s)$ and $\hat{L}=2(d)$ bosons are retained. The model has a $U(6)$ group structure and three possible dynamical symmetry limits representing welldefined nuclear phases: the $U(5)$ symmetry for vibrational nuclei, the $O(6)$ symmetry for $\gamma$-unstable nuclei, and the $S U(3)$ symmetry for axially deformed nuclei. In each of the three limits the Hamiltonian can be expressed in terms of the Casimir operators of the group decomposition chain. The three limits are then exactly solvable with analytic expressions for the eigenstates.

The transition from $U(5)$ to $O(6)$ can be modelled by a boson paring Hamiltonian of the form [44]

$$
H_{\mathrm{IBM} 1}=x\left(n_{d}-n_{s}\right)+\frac{1-x}{N} \sum_{\mu=-2}^{2}\left(d_{\mu}^{\dagger} d_{\bar{\mu}}^{\dagger} s s+s^{\dagger} s^{\dagger} d_{\bar{\mu}} d_{\mu}\right),
$$

where $N$ is the total number of bosons and $x$ is a parameter that interpolates between the linear Casimir operator of $U(5)$, for $x=1$, and the quadratic Casimir operator of $O(6)$, for $x=0$. The Hamiltonian $H_{\mathrm{IBM} 1}$ can be derived from Eq. (95) by making the following identifications: $d=b, s=a, \Omega_{d}=5, \Omega_{s}=1, \varepsilon=2 x, w=0$, and $v=\frac{(1-x)}{N}$.

The transition from the spherical vibrational phase $(U(5))$ to the $\gamma$-unstable deformed phase was studied within the integrable model described by the Hamiltonian of Eq. (99) [45]. It was found a second order quantum phase transition for a critical value of the control parameter $x$. In fact this is a unique point of second order phase transitions in the complete 
parameter space of the most general IBM Hamiltonian. The second-order character of the transition is related to quantum integrability. The ground state eigenvalue of $H_{\mathrm{IBM} 1}$ is an analytic function of the control parameter $x$ and, though level crossings are allowed due to quantum integrability, there are no level crossings in the low-energy spectrum.

\subsubsection{The Lipkin-Meshkov-Glick model}

The LMG model has been extensively used for decades to simulate the phase transition from spherical to deformed shapes in finite nuclei. As introduced in Section 5.2, it is a schematic model describing the scattering of particle-hole pairs between two shells of different parity $\sigma$. Though it was known for a long time that the model was quantum integrable, some analytic solutions were found only quite recently [46] using the algebraic Bethe ansatz, after having mapped the model onto a Schwinger-boson representation. Here we will show that the LMG model is exactly solvable: after a Schwinger-boson representation of angular momentum operators, we will map the LMG model onto the two-boson integrable Hamiltonians of Eq. (95).

In the Schwinger mapping of the $s u(2)$ algebra the generators are expressed in terms of two bosons $a$ and $b$ as

$$
S^{+}=b^{\dagger} a=\left(S^{-}\right)^{\dagger}, \quad S^{z}=\frac{1}{2}\left(b^{\dagger} b-a^{\dagger} a\right)=\frac{1}{2}\left(n_{b}-n_{a}\right),
$$

with the constraint

$$
2 \mathrm{~S}=b^{\dagger} b+a^{\dagger} a=n_{b}+n_{a} .
$$

Inserting Eq. (100) into Eq. (79) we obtain a Schwinger-boson representation of the LMG Hamiltonian

$$
H_{\mathrm{LMG}}=\frac{W}{N} \mathrm{~S}+\frac{\varepsilon}{2}\left(n_{b}-n_{a}\right)+\frac{V}{2 N}\left(b^{\dagger} b^{\dagger} a a+a^{\dagger} a^{\dagger} b b\right)+\frac{W}{N} n_{b} n_{a} .
$$

We then recover the two-boson exactly solvable Hamiltonian of Eq. (95) with $\Omega_{b}=$ $\Omega_{a}=1, v=V / 2 N$ and $w=W / N$. In particular, the (unnormalized) eigenvectors and eigenvalues of $H_{\mathrm{LMG}}$ are

$$
\begin{aligned}
& |\Psi\rangle_{\mathrm{LMG}}=\prod_{\ell=1}^{M}\left(\frac{a^{\dagger} a^{\dagger}}{E_{\ell}+\eta}+\frac{b^{\dagger} b^{\dagger}}{E_{\ell}-\eta}\right)|v\rangle, \\
& E_{\mathrm{LMG}}=E_{\mathrm{B} 2}-\frac{w}{4},
\end{aligned}
$$

where $E_{\mathrm{B} 2}$ is given in Eq. (98), and the spectral parameters $E_{\ell}$ satisfy Eq. (97). Notice that, for each sector $S$, the number of bosons that enter in the expression for $|\Psi\rangle_{L M G}$ is constrained to be $2 \mathrm{~S}=n_{a}+n_{b}$.

We would like to emphasize that our expressions are compact forms valid for any arbitrary set of parameters in the Hamiltonian. In particular, they are valid for the parameter range $W^{2}<V^{2}$, which corresponds to the solutions not found in Ref. [46]. Since we have shown (using the weakly-interacting limit solutions) that this Bethe ansatz covers all possible eigenstates, it implies that the LMG is exactly solvable. 


\subsubsection{Two coupled Bose-Einstein condensates}

The Josephson effect, predicted more than forty years ago [47], describes pair tunnelling between two superconductors through an insulating junction. An analogous effect can be realized with trapped ultracold bosonic gases in two different ways. In the first setup, two atomic condensates in the same atomic state are separated by a controllable potential barrier. In the second setup, atoms are condensed in two overlapping hyperfine states with an exchange mixing interaction. Both systems are described by the Hamiltonian [14]

$$
H_{\mathrm{J}}=-\frac{E_{\mathrm{J}}}{N}\left(c^{\dagger} d+d^{\dagger} c\right)+\frac{E_{c}}{4}\left(c^{\dagger} c c^{\dagger} c+d^{\dagger} d d^{\dagger} d\right),
$$

where $c^{\dagger}$ and $d^{\dagger}$ are left or right trap boson creation operators, or they create bosons in two different hyperfine states, depending upon the particular setup. $E_{\mathrm{J}}$ is the Josephson coupling exchanging bosons between the two states, and $E_{c}$ is the charging energy. This Hamiltonian has been recently exactly solved using the algebraic Bethe ansatz [48]. In fact, it is no more than another form of the two-level boson pairing Hamiltonian of Eq. (95). It can be easily recast in the two-level form after performing the unitary canonical transformation

$$
c=\frac{1}{\sqrt{2}}(a-i b), \quad d=\frac{1}{\sqrt{2}}(a+i b) .
$$

Eliminating irrelevant constant terms, the Josephson Hamiltonian can be rewritten as

$$
H_{\mathrm{J}}=\frac{E_{\mathrm{J}}}{N}\left(n_{b}-n_{a}\right)-\frac{E_{c}}{8}\left(b^{\dagger} b^{\dagger} a a+a^{\dagger} a^{\dagger} b b-2 n_{b} n_{a}\right),
$$

which can be easily related to the LMG Hamiltonian, $H_{\mathrm{LMG}}$, by choosing $\varepsilon=2 E_{\mathrm{J}} / N, V=$ $-N E_{c} / 4=-W$. Therefore, the physics of the two coupled BECs is completely analogous to that of the LMG model.

\section{Mixing realizations and representations of the Gaudin field operators}

We have already mentioned that our scheme for generating completely integrable models relies on finding different representations of the GGA. So far, we have simply concentrated on exactly solvable models where every single $s u(2)$ or $s u(1,1)$ generator labelled by an index in the set $\mathcal{T}$ is equivalently represented. We still have the freedom to mix the representations of these generators and develop new exactly-solvable model Hamiltonians. The fact that the two su(2) realizations of Eqs. (81) and (82) are mutually commuting was also noted in [37]. They called them the spin and the charge realizations, respectively. This property implies that the elements of the spin and charge $s u(2)$ algebras act on orthogonal Hilbert spaces, allowing to define an integrable RG model in each space separately. Thus, the following Hamiltonian [37] was proposed to study the interplay between pairing correlations and spin-exchange interactions

$$
H_{\mathrm{ch}-S}=\sum_{\mathbf{i}} \varepsilon_{\mathbf{i}} \tau_{\mathbf{i}}^{z}+\frac{\tilde{g}}{2 N} \sum_{\mathbf{i}, \mathbf{j}(\mathbf{i} \neq \mathbf{j})}\left(\tilde{X}_{\mathbf{i j}}\left(\tau_{\mathbf{i}}^{+} \tau_{\mathbf{j}}^{-}+\tau_{\mathbf{i}}^{-} \tau_{\mathbf{j}}^{+}\right)+2 \tilde{Z}_{\mathbf{i j}} \tau_{\mathbf{i}}^{z} \tau_{\mathbf{j}}^{z}+W_{\mathbf{i j}} S_{\mathbf{i}} \cdot S_{\mathbf{j}}\right)
$$


where we can recognize the most general integrable pairing Hamiltonian, Eq. (46), with an additional spin-exchange interaction, which is also integrable if the matrix $W$ is derived from the rational Gaudin family as $W_{\mathbf{i j}}=\tilde{X}_{\mathbf{i j}}^{\prime}$. The use of the rational family assures the conservation of the total spin quantum number as well as the third component of the total spin. The model Hamiltonian $H_{\mathrm{ch}-\mathrm{S}}$ can still accommodate a linear term in the spin variables representing a nonuniform magnetic field, or even more general $X X Z$ Gaudin models can be implemented in the spin space at the cost of breaking the spin rotational symmetry.

A numerical study of the interplay between pairing and exchange interactions in small metallic dots has been carried out in [49] for systems with up to 30 levels. Even though the model used was fully integrable, the numerical results were mostly obtained by large scale diagonalization methods due to the complexity in solving the two sets of coupled Richardson's equations. The recently developed numerical techniques [32] to solve efficiently these set of nonlinear equations may help to extend these studies to larger grains.

The space orthogonality between the two $s u(2)$ fermionic realizations can also be exploited by defining different integrable models in the charge and spin sectors. For instance, it would be possible to mix a RG integrable Hamiltonian in the charge space with a Heisenberg or Haldane-Shastry model in the spin space. By mixing different realizations of the GGA, one can generate spin-fermion, spin-boson, or simply spin models with spins belonging to different irreducible representations. It turns out that this mixing-representations scheme might be useful to study decoherence and dynamic phenomena in open quantum systems where some degrees of freedom correspond to the system while the others (coupled in a particular way to the system) represent the thermal bath. In the following we illustrate these ideas starting with the generalized Dicke (GD) model Hamiltonian.

\subsection{Generalized Dicke models}

Generalizations of the Dicke model, solved by the algebraic Bethe ansatz, have been reported in [50,51]. Here, following the GGA approach, we will present a different generalization of the Dicke model [52].

Starting from the $X X Z \mathrm{RG}$ models, we replace one of the $s u(2)$ copies by a single boson satisfying the Heisenberg-Weyl algebra. This procedure can be rigorously followed by expressing the $s u(2)$ generators in the Holstein-Primakoff representation as

$$
S_{0}^{+}=\sqrt{2 \mathrm{~S}_{0}} b^{\dagger} \sqrt{1-\frac{b^{\dagger} b}{2 \mathrm{~S}_{0}}}=\left(S_{0}^{-}\right)^{\dagger}, \quad S_{0}^{z}=-\mathrm{S}_{0}+b^{\dagger} b,
$$

where we have distinguished the particular copy of $s u(2)$ by the label $0, b^{\dagger}(b)$ is the creation (annihilation) operator of a boson with $\left[b, b^{\dagger}\right]=1$, and $\mathrm{S}_{0}$ is the magnitude of the spin of this particular representation. Since the RG model is integrable for arbitrary spin values we can then analyze the limit $\mathrm{S}_{0} \rightarrow \infty$, which implies the replacement

$$
S_{0}^{+}=\sqrt{2 S_{0}} b^{\dagger}, \quad S_{0}^{-}=\sqrt{2 S_{0}} b .
$$


We will skip here the derivation of the new class of integrable spin-boson models that can be found in [52] and present the final form of the integrals of motion

$$
\begin{aligned}
R_{0}= & \omega b^{\dagger} b+2 \sum_{\mathbf{j}} \varepsilon_{\mathbf{j}} S_{\mathbf{j}}^{z}+V \sum_{\mathbf{j}}\left(b^{\dagger} S_{\mathbf{j}}^{-}+b S_{\mathbf{j}}^{+}\right), \\
R_{\mathbf{i}}= & \omega S_{\mathbf{i}}^{z}+\frac{V^{2}}{2} \sum_{\mathbf{j} \neq \mathbf{i})} \frac{1}{\varepsilon_{\mathbf{i}}-\varepsilon_{\mathbf{j}}}\left[S_{\mathbf{i}}^{+} S_{\mathbf{j}}^{-}+S_{\mathbf{i}}^{-} S_{\mathbf{j}}^{+}+2 S_{\mathbf{i}}^{z} S_{\mathbf{j}}^{z}\right] \\
& -V\left(b^{\dagger} S_{\mathbf{i}}^{-}+b S_{\mathbf{i}}^{+}\right)-2 \varepsilon_{\mathbf{i}} S_{\mathbf{i}}^{z} .
\end{aligned}
$$

It can be readily verified that the set of operators of Eqs. (109) are Hermitian, independent, and mutually commuting. Therefore, they constitute a new class of integrable spin-boson models. Though they have been derived from the trigonometric family of the RG models, the set of $L$ operators $R_{\mathbf{i}}$ are identical to the rational family of RG models, except for the last two terms of $s u(2)$ which are essential for ensuring the commutation with the new bosonic integral of motion $R_{0}$.

Any function of these operators defines an integrable Hamiltonian. In particular, we can recognize $R_{0}$ as a Dicke Hamiltonian describing the interactions of a multi-atom system with a single-mode radiation field. Moreover, a linear combination involving the whole set of integrals of motion, Eqs. (109), gives rise to more general integrable spin-boson Hamiltonians.

Richardson's ansatz for the common eigenstates of the integrals of motion is

$$
|\Psi\rangle=\prod_{\alpha=1}^{M}\left(b^{\dagger}+\sum_{\mathbf{i}=1}^{L} \frac{V}{y_{\alpha}-\varepsilon_{\mathbf{i}}} S_{\mathbf{i}}^{+}\right)|0\rangle,
$$

where $L$ is the total number of $s u(2)$ spins and $M$ the total spin and third component of the system. The $M$ spectral parameters $y_{\alpha}$ are particular solutions of the set of $M$ of nonlinear coupled Richardson's equations

$$
\frac{\omega}{2 V}-\frac{1}{2 V} y_{\alpha}-\frac{V}{2} \sum_{\mathbf{i}} \frac{\mathbf{S}_{\mathbf{i}}}{2 \varepsilon_{\mathbf{i}}-y_{\alpha}}-V \sum_{\beta(\neq \alpha)} \frac{1}{y_{\alpha}-y_{\beta}}=0 .
$$

The corresponding expressions for the eigenvalues of Eqs. (109) are

$$
\begin{aligned}
& r_{0}=\sum_{\alpha} y_{\alpha}-\sum_{\mathbf{j}} \mathrm{S}_{\mathbf{j}} \varepsilon_{\mathbf{j}}, \\
& r_{\mathbf{i}}=-\frac{\mathrm{S}_{\mathbf{i}}}{2}\left\{\omega+2 \varepsilon_{\mathbf{i}}-\frac{V^{2}}{2} \sum_{\mathbf{j}(\neq \mathbf{i})} \frac{\mathrm{S}_{\mathbf{j}}}{\varepsilon_{\mathbf{i}}-\varepsilon_{\mathbf{j}}}+2 V^{2} \sum_{\alpha} \frac{1}{y_{\alpha}-2 \varepsilon_{\mathbf{i}}}\right\} .
\end{aligned}
$$

A similar treatment can be developed for the $s u(1,1)$ case [52]. This case is relevant for representing the interaction between bosonic atoms and dimer molecules. Note that the appropriate Holstein-Primakoff representation for the $s u(1,1)$ algebra is

$$
K_{0}^{+}=\sqrt{2 \mathrm{~K}_{0}} b^{\dagger} \sqrt{1+\frac{b^{\dagger} b}{2 \mathrm{~K}_{0}}}=\left(K_{0}^{-}\right)^{\dagger}, \quad K_{0}^{z}=\mathrm{K}_{0}+b^{\dagger} b .
$$




\subsection{An exactly-solvable Kondo-like impurity model}

The effect of magnetic impurities in metals has been a subject of intense debate since the early 1930s. In 1964 Kondo [53] made significant progress by providing an explanation to the problem of the resistance minimum (as a function of temperature) in some metals such as $\mathrm{Au}$. He recognized that the interaction of a single magnetic impurity with the conduction electrons is well represented by the $s-d$ exchange Hamiltonian

$$
H_{\mathrm{Sd}}=\sum_{\mathbf{k}, \mathbf{k}^{\prime}} J_{\mathbf{k} \mathbf{k}^{\prime}}\left(S^{-} c_{\mathbf{k} \uparrow}^{\dagger} c_{\mathbf{k}^{\prime} \downarrow}+S^{+} c_{\mathbf{k} \downarrow}^{\dagger} c_{\mathbf{k}^{\prime} \uparrow}+S^{z}\left(c_{\mathbf{k} \uparrow}^{\dagger} c_{\mathbf{k}^{\prime} \uparrow}-c_{\mathbf{k} \downarrow}^{\dagger} c_{\mathbf{k}^{\prime} \downarrow}\right)\right),
$$

where $S^{z}, S^{ \pm}$are the spin operators representing the localized moment of magnitude $\mathrm{S}$, while $c_{\mathbf{k} \sigma}^{\dagger}$ creates a conduction electron with momentum $\mathbf{k}$ and spin projection $\sigma$. This type of interaction is characterized by terms in which the spin of the electron is flipped upon scattering with the impurity and are essential to understand the logarithmic contribution to the resistivity, and thus, its minimum. The simplest Hamiltonian representing the interaction of a localized moment with a band of itinerant electrons is the Kondo impurity model

$$
H_{\mathrm{K}}=\sum_{\mathbf{k}, \sigma} \varepsilon_{\mathbf{k}} n_{\mathbf{k} \sigma}+H_{\mathrm{sd}}
$$

It is important to emphasize that the case $\mathrm{S}=1 / 2, J_{\mathbf{k k}^{\prime}}=J / N$, and linear (relativistic) dispersion has been exactly solved by Andrei and Weigmann using the Bethe ansatz [54].

To find a new exactly-solvable single impurity Kondo-like model let us consider the RG constants of motion, where the localized spin of magnitude $S$ is singled out

$$
R_{0}=B S^{z}+\sum_{\mathbf{j}}\left(X_{0 \mathbf{j}}\left(S^{-} S_{\mathbf{j}}^{+}+S^{+} S_{\mathbf{j}}^{-}\right)+2 Z_{0 \mathbf{j}} S^{z} S_{\mathbf{j}}^{z}\right),
$$

with electron spins given by

$$
S_{\mathbf{j}}^{+}=c_{\mathbf{j} \uparrow}^{\dagger} c_{\mathbf{j} \downarrow}=\left(S_{\mathbf{j}}^{-}\right)^{\dagger}, \quad S_{\mathbf{j}}^{z}=\frac{1}{2}\left(n_{\mathbf{j} \uparrow}-n_{\mathbf{j} \downarrow}\right) .
$$

As mentioned above the commuting $s u(2)$ algebra

$$
\tau_{\mathbf{j}}^{+}=c_{\mathbf{j} \uparrow}^{\dagger} c_{\mathbf{j} \downarrow}^{\dagger}=\left(\tau_{\mathbf{j}}^{-}\right)^{\dagger}, \quad \tau_{\mathbf{j}}^{z}=\frac{1}{2}\left(n_{\mathbf{j} \uparrow}+n_{\mathbf{j} \downarrow}-1\right),
$$

is a gauge symmetry of $R_{0}$, thus one can write down the following exactly-solvable Gaudin model

$$
\begin{aligned}
H_{\mathrm{Gl}}= & 2 \sum_{\mathbf{j}} \varepsilon_{\mathbf{j}} \tau_{\mathbf{j}}^{z}+R_{0} \\
= & \sum_{\mathbf{j}} \varepsilon_{\mathbf{j}}\left(n_{\mathbf{j} \uparrow}+n_{\mathbf{j} \downarrow}-1\right)+B S^{z} \\
& +\sum_{\mathbf{k}, \mathbf{k}^{\prime}}\left(J_{\mathbf{k k}^{\prime}}^{\perp}\left(S^{-} c_{\mathbf{k} \uparrow}^{\dagger} c_{\mathbf{k}^{\prime} \downarrow}+S^{+} c_{\mathbf{k} \downarrow}^{\dagger} c_{\mathbf{k}^{\prime} \uparrow}\right)+J_{\mathbf{k} \mathbf{k}^{\prime}}^{\|} S^{z}\left(c_{\mathbf{k} \uparrow}^{\dagger} c_{\mathbf{k}^{\prime} \uparrow}-c_{\mathbf{k} \downarrow}^{\dagger} c_{\mathbf{k}^{\prime} \downarrow}\right)\right),
\end{aligned}
$$


where

$$
J_{\mathbf{k} \mathbf{k}^{\prime}}^{\perp(\|)}=\frac{1}{N} \sum_{\mathbf{j}} e^{i\left(\mathbf{k}-\mathbf{k}^{\prime}\right) \cdot \mathbf{r}_{\mathbf{j}}} X_{0 \mathbf{j}}\left(Z_{0 \mathbf{j}}\right), \quad c_{\mathbf{j} \sigma}^{\dagger}=\frac{1}{\sqrt{N}} \sum_{\mathbf{k}} e^{i \mathbf{k} \cdot \mathbf{r}_{\mathbf{j}}} c_{\mathbf{k} \sigma}^{\dagger}
$$

is the Fourier-transformed electron operator.

To derive an exactly-solvable Kondo-like Hamiltonian one considers the particular case $J_{\mathbf{k} \mathbf{k}^{\prime}}^{\perp}=J X_{0 \mathbf{k}} \delta_{\mathbf{k} \mathbf{k}^{\prime}}$ and $J_{\mathbf{k} \mathbf{k}^{\prime}}^{\|}=J Z_{0 \mathbf{k}} \delta_{\mathbf{k k}^{\prime}}$ in $H_{\mathrm{sd}}$, and adds the conduction band term

$$
\begin{aligned}
H_{\mathrm{GK}}= & \sum_{\mathbf{k}, \sigma} \varepsilon_{\mathbf{k}} n_{\mathbf{k} \sigma}+B S^{z} \\
& +J \sum_{\mathbf{k}}\left(X_{0 \mathbf{k}}\left(S^{-} c_{\mathbf{k} \uparrow}^{\dagger} c_{\mathbf{k} \downarrow}+S^{+} c_{\mathbf{k} \downarrow}^{\dagger} c_{\mathbf{k} \uparrow}\right)+Z_{0 \mathbf{k}} S^{z}\left(c_{\mathbf{k} \uparrow}^{\dagger} c_{\mathbf{k} \uparrow}-c_{\mathbf{k} \downarrow}^{\dagger} c_{\mathbf{k} \downarrow}\right)\right),
\end{aligned}
$$

since in this case $\tau_{\mathbf{k}}^{z}$ commutes with $R_{0}$. (Notice that the magnitude of the localized spin is not restricted to $S=1 / 2$.) Both, a minimum in the electrical resistivity, and the formation of a singlet resonance state characterize the Kondo physics. Clearly, since $H_{\mathrm{GK}}$ is translationally invariant the impurity contribution to the charge resistivity is zero. However, using this model one may address the fundamental issue of the formation of the singlet state, writing down a many-body state that captures the essence of the Kondo problem.

The (unnormalized) $N$-particles eigenstates of $H_{\mathrm{GK}}$ are given by

$$
|\Psi\rangle=\prod_{\ell=1}^{M}\left(X_{\ell 0} S^{+}+\sum_{\mathbf{k}} X_{\ell \mathbf{k}} c_{\mathbf{k} \uparrow}^{\dagger} c_{\mathbf{k} \downarrow}\right)|\mathrm{FS}\rangle,
$$

where $|\mathrm{FS}\rangle$ is the tensor product of the state $|v\rangle=\left|\nu_{\mathbf{k}_{1}} \cdots v_{\mathbf{k}_{j}} \cdots\right\rangle$ (of $v=\sum_{\mathbf{k}} \nu_{\mathbf{k}}$ paired $\left(v_{\mathbf{k}}=2\right)$ fermions) with the remaining $N-v$ fermions in a ferromagnetic state, and the lowest-weight spin state $|0\rangle_{S}$

$$
|\mathrm{FS}\rangle=|v\rangle \otimes \underbrace{\prod_{\mathbf{k}} c_{\mathbf{k} \downarrow}^{\dagger}|0\rangle}_{N-v} \otimes|0\rangle_{S},
$$

while $E_{\ell}$ 's satisfy the Bethe equations

$$
\frac{B}{2 J}=d_{0} Z_{0 \ell}+\sum_{\mathbf{k}} d_{\mathbf{k}} Z_{\mathbf{k} \ell}+\sum_{n(\neq \ell)=1}^{M} Z_{n \ell}, \quad \ell=1, \ldots, M,
$$

and the energy eigenvalues are given by $\left(\nu_{\mathbf{k}}=0,1,2\right)$

$$
E=\sum_{\mathbf{k}} \varepsilon_{\mathbf{k}} v_{\mathbf{k}}+d_{0}\left(B+2 J \sum_{\ell} Z_{0 \ell}+2 J \sum_{\mathbf{k}} d_{\mathbf{k}} Z_{0 \mathbf{k}}\right) .
$$

The case of zero magnetic field $(B=0)$ corresponds to the Gaudin magnet.

Any set of parameters $X_{0 \mathbf{k}}, Z_{0 \mathbf{k}}$ for which $X_{0 \mathbf{k}}^{2}-Z_{0 \mathbf{k}}^{2}=\Gamma$ (see Eq. (12)) leads to an integrable exactly-solvable model. Particularly, the case of a spin-isotropic exchange interaction, i.e., $X_{0 \mathbf{k}}=Z_{0 \mathbf{k}}=1 /\left(\eta_{0}-\eta_{\mathbf{k}}\right)$, is of interest since the total spin is a good quantum 
number. It is easy to see that if the exchange coupling is antiferromagnetic (e.g., $J>0$, $\eta_{0}>\eta_{\mathbf{k}}$, and $B$ smaller than a critical value) a singlet Kondo many-body state emerges in the problem. The emergence of this state is formally connected to BCS superconductivity, and the connection is established through Bethe's equations. In this way, for $M>1$, we are rigorously connecting the Kondo resonance with the Cooper resonance problems.

\subsection{Exactly-solvable spin-boson models}

The study of spin-boson systems, i.e., a single spin of magnitude $S$ linearly coupled to a thermal bath represented by a set of harmonic oscillators, is of particular interest in the theory of open quantum systems. These systems display important features of decoherence, that is the dynamical loss of quantum coherence because of the environment. In this section we will describe two interesting spin-boson models that are exactly-solvable.

Let us start from the constant of motion $R_{0}$ of Eq. (114) and add to it the total magnetization symmetry

$$
H_{\mathrm{sb} 1}=(B+\omega) S^{z}+\sum_{\mathbf{j}} X_{0 \mathbf{j}}\left(S^{-} S_{\mathbf{j}}^{+}+S^{+} S_{\mathbf{j}}^{-}\right)+2 \sum_{\mathbf{j}} Z_{0 \mathbf{j}} S^{z} S_{\mathbf{j}}^{z}+\omega \sum_{\mathbf{j}} S_{\mathbf{j}}^{z} .
$$

Following a similar procedure to the one illustrated in Section 7.1 we represent the $s u(2)$ spins $S_{\mathbf{j}}$ (in the limit $\mathrm{S}_{\mathrm{b}} \rightarrow \infty$ ) as

$$
S_{\mathbf{j}}^{+}=\sqrt{2 S_{\mathrm{b}}} b_{\mathbf{j}}^{\dagger}, \quad S_{\mathbf{j}}^{-}=\sqrt{2 \mathrm{~S}_{\mathrm{b}}} b_{\mathbf{j}}, \quad S_{\mathbf{j}}^{z}=-\mathrm{S}_{\mathrm{b}}+b_{\mathbf{j}}^{\dagger} b_{\mathbf{j}},
$$

and choose

$$
X_{0 \mathbf{j}}=g\left(1+\frac{\varepsilon_{\mathbf{j}}^{2}}{4 \mathrm{~S}_{\mathrm{b}}}\right), \quad Z_{0 \mathbf{j}}=-g \sqrt{\frac{1}{2 \mathrm{~S}_{\mathrm{b}}}} \varepsilon_{\mathbf{j}},
$$

with the resulting Hamiltonian (up to irrelevant constants)

$$
H_{\mathrm{sb} 1}=\bar{B} S^{z}+\sum_{\mathbf{j}} \omega b_{\mathbf{j}}^{\dagger} b_{\mathbf{j}}+V \sum_{\mathbf{j}}\left(S^{-} b_{\mathbf{j}}^{\dagger}+S^{+} b_{\mathbf{j}}\right),
$$

where $V=g \sqrt{2 \mathrm{~S}_{\mathrm{b}}}$, and $\bar{B}=\tilde{B}+\omega$, with $\tilde{B}=B+V \sum_{\mathbf{j}} \varepsilon_{\mathbf{j}}$. Notice that the localized spin $S$ may have arbitrary magnitude $S$. This model is known to be trivially solvable. Indeed, it is a particular case of the Fröhlich-like Hamiltonian describing a spin coupled to longitudinal optical phonons

$$
H_{\mathrm{sb} 1}=\bar{B} S^{z}+\sum_{\mathbf{j}} \omega b_{\mathbf{j}}^{\dagger} b_{\mathbf{j}}+\sum_{\mathbf{j}}\left(V_{\mathbf{j}}^{*} S^{-} b_{\mathbf{j}}^{\dagger}+V_{\mathbf{j}} S^{+} b_{\mathbf{j}}\right),
$$

which is diagonalized by first performing a unitary canonical mapping to new bosonic modes $a_{\mathbf{j}}$

$$
\left(\begin{array}{c}
a_{1} \\
a_{2} \\
a_{3} \\
\vdots \\
a_{N}
\end{array}\right)=\left(\begin{array}{ccccc}
v_{1} & v_{2} & v_{3} & \cdots & v_{N} \\
v_{2}^{*} & A_{22} & A_{23} & \cdots & A_{2 N} \\
v_{3}^{*} & A_{32} & A_{33} & \cdots & A_{3 N} \\
\vdots & \vdots & \vdots & \cdots & \vdots \\
v_{N}^{*} & A_{N 2} & A_{N 3} & \cdots & A_{N N}
\end{array}\right)\left(\begin{array}{c}
b_{1} \\
b_{2} \\
b_{3} \\
\vdots \\
b_{N}
\end{array}\right),
$$


where $\left(\Lambda=\sqrt{\sum_{\mathbf{j}}\left|V_{\mathbf{j}}\right|^{2}}\right)$

$$
v_{\mathbf{i}}=\frac{V_{\mathbf{i}}}{\Lambda}, \quad A_{\mathbf{i i}}=\frac{\left|v_{\mathbf{i}}\right|^{2}-1-v_{1}^{*}}{1+v_{1}}, \quad A_{\mathbf{i j}}=\frac{v_{\mathbf{i}}^{*} v_{\mathbf{j}}}{1+v_{1}},
$$

yielding the Hamiltonian

$$
H_{\mathrm{sb} 1}=\bar{B} S^{z}+\sum_{\mathbf{j}} \omega a_{\mathbf{j}}^{\dagger} a_{\mathbf{j}}+\Lambda\left(S^{-} a_{1}^{\dagger}+S^{+} a_{1}\right),
$$

representing, in the new basis, optical phonons effectively interacting with a single spin through a single mode.

A more elaborate spin-boson model can be realized using the other constants of motion. In this way the following model Hamiltonian results

$$
H_{\mathrm{sb} 2}=\tilde{B} S^{z}+\sum_{\mathbf{i}, \mathbf{j}} t_{\mathbf{i j}}\left(b_{\mathbf{i}}^{\dagger} b_{\mathbf{j}}-b_{\mathbf{i}}^{\dagger} b_{\mathbf{i}}\right)+V \sum_{\mathbf{j}}\left(S^{-} b_{\mathbf{j}}^{\dagger}+S^{+} b_{\mathbf{j}}\right),
$$

where $t_{\mathbf{i j}}=\frac{x_{\mathbf{i}}-x_{\mathbf{j}}}{\varepsilon_{\mathbf{i}}-\varepsilon_{\mathbf{j}}}$, with $\varepsilon_{\mathbf{i}}$ representing the parameters defining the model, Eq. (125), and $x_{\mathbf{i}}$ the coefficients in the linear combination of the integrals of motion.

The eigenstates of $H_{\mathrm{sb} 2}$ are

$$
|\Psi\rangle=\prod_{\alpha}\left(y_{\alpha} S^{+}+\sum_{\mathbf{j}} b_{\mathbf{j}}^{\dagger}\right)|0\rangle,
$$

where the spectral parameters $y_{\alpha}$ are the solutions of the Bethe equations

$$
1-\frac{\Omega_{0}}{2} V y_{\alpha}+V \sum_{\mathbf{j}} \frac{1+\varepsilon_{\mathbf{j}} y_{\alpha}}{y_{\alpha}}-2 V \sum_{\beta(\neq \alpha)} \frac{y_{\alpha} y_{\beta}}{y_{\beta}-y_{\alpha}}=0
$$

and $\Omega_{0}=2 \mathrm{~S}+1$. The corresponding eigenvalues are

$$
E_{\mathrm{sb} 2}=\frac{\Omega_{0}}{2} V \sum_{\alpha} y_{\alpha}-\frac{G}{2} \sum_{j \neq i} \frac{\left(x_{i}-x_{j}\right) \varepsilon_{i} \varepsilon_{j}}{\varepsilon_{i}-\varepsilon_{j}} .
$$

\section{Differential operator realizations: Schrödinger-Gaudin operators}

So far we concentrated on discrete representations which led to various exactly solvable lattice models. However, it is well-known that it is possible to realize representations of Lie algebras in the form of differential operators. As we will see in this section these representations will lead to models in the continuum. Basically we will generalize the work pioneered by Ushveridze and others [3].

Out of the many applications one can foresee we will concentrate on a single problem. The problem consists of mapping exactly-solvable lattice models to their equivalent in the continuum. Clearly, in general, the full spectrum of the lattice will be embedded in the spectrum of the continuum equivalent. For the sake of simplicity we will use the $\bigoplus_{\mathbf{l}} \operatorname{su}(2)$ representation. 
To illustrate the procedure we will use the BCS pairing lattice model of Eq. (63) which, after realizing about its underlying algebraic structure, reads

$$
H_{\mathrm{BCS}}=2 \sum_{\mathbf{k}} \varepsilon_{\mathbf{k}} \tau_{\mathbf{k}}^{z}+g \sum_{\mathbf{k} \mathbf{k}^{\prime}} \tau_{\mathbf{k}}^{+} \tau_{\mathbf{k}^{\prime}}^{-}
$$

We wonder what the many-body problem in configuration space (a continuous manifold), to which it maps onto, is. To this end, we will consider the corresponding $s u(2)$ Lie algebra of differential operators in the tensor product representation

$$
\begin{aligned}
\tau_{\mathbf{k}}^{+} & =z_{\mathbf{k}}, \\
\tau_{\mathbf{k}}^{-} & =-z_{\mathbf{k}} \partial_{z_{\mathbf{k}}}^{2}+2 \mathrm{~S}_{\mathbf{k}} \partial_{z_{\mathbf{k}}}, \\
\tau_{\mathbf{k}}^{z} & =-\mathrm{S}_{\mathbf{k}}+z_{\mathbf{k}} \partial_{z_{\mathbf{k}}},
\end{aligned}
$$

with Casimir operator $\tau_{\mathbf{k}}^{2}=\frac{1}{2}\left(\tau_{\mathbf{k}}^{+} \tau_{\mathbf{k}}^{-}+\tau_{\mathbf{k}}^{-} \tau_{\mathbf{k}}^{+}\right)+\tau_{\mathbf{k}}^{z} \tau_{\mathbf{k}}^{z}=\mathrm{S}_{\mathbf{k}}\left(\mathrm{S}_{\mathbf{k}}+1\right)$ and where it is assumed that the differential generators act on polynomials in the variables $z_{\mathbf{k}}$ of maximum order $2 S_{\mathbf{k}}$. In other words, the order of the polynomials depends upon the value $S_{\mathbf{k}}$ of the spin irreducible representation. In this differential operator representation Eq. (132) can be written

$$
\begin{aligned}
& H_{\mathrm{BCS}}=2 \sum_{\mathbf{k}} \varepsilon_{\mathbf{k}}\left(-\mathrm{S}_{\mathbf{k}}+z_{\mathbf{k}} \partial_{z_{\mathbf{k}}}\right)+g \varphi\left(\left\{z_{\mathbf{k}}\right\}\right) \sum_{\mathbf{k}}\left(-z_{\mathbf{k}} \partial_{z_{\mathbf{k}}}^{2}+2 \mathrm{~S}_{\mathbf{k}} \partial_{z_{\mathbf{k}}}\right), \\
& H_{\mathrm{BCS}}=\bar{E}+g \varphi\left(\left\{z_{\mathbf{k}}\right\}\right) \sum_{\mathbf{k}} \frac{1}{4}\left[-i \partial_{x_{\mathbf{k}}}+A\right]^{2}+V\left(\left\{x_{\mathbf{k}}\right\}\right),
\end{aligned}
$$

where $\bar{E}=-2 \sum_{\mathbf{k}} \varepsilon_{\mathbf{k}} \mathbf{S}_{\mathbf{k}}, \varphi\left(\left\{z_{\mathbf{k}}\right\}\right)=\sum_{\mathbf{k}} z_{\mathbf{k}}, z_{\mathbf{k}}=x_{\mathbf{k}}^{2}$,

$$
A=2 i\left(\frac{\left(\mathrm{S}_{\mathbf{k}}+\frac{1}{4}\right)}{x_{\mathbf{k}}}+\frac{\varepsilon_{\mathbf{k}} x_{\mathbf{k}}}{g \varphi\left(\left\{z_{\mathbf{k}}\right\}\right)}\right), \quad 4 V\left(\left\{x_{\mathbf{k}}\right\}\right)=i\left(\partial_{x_{\mathbf{k}}} A\right)-A^{2} .
$$

Notice that, in this language, $H_{\mathrm{BCS}}$ represents a many-particle system in a gauge field subjected to a potential $V$.

In the case $\mathbf{S}_{\mathbf{k}}=1 / 2$, for all $\mathbf{k}$ 's (no unpair single-particle states)

$$
|\uparrow\rangle_{\mathbf{k}} \rightarrow z_{\mathbf{k}}, \quad|\downarrow\rangle_{\mathbf{k}} \rightarrow 1
$$

and the representation space includes polynomials of degree at most 1 in each variable. In the following we will simply concentrate on this case which corresponds to degeneracy $\Omega_{\mathbf{k}}=2$. It is straightforward to prove that the ansatz wave function

$$
\Psi^{M}\left(\left\{s_{\mathbf{k}}\right\}\right)=\sum_{\mathbf{k}_{1}, \ldots, \mathbf{k}_{M}}^{\prime} s_{\mathbf{k}_{1}} s_{\mathbf{k}_{2}} \cdots s_{\mathbf{k}_{M}},
$$

where the prime in the sum means that $\mathbf{k}_{1} \neq \mathbf{k}_{2} \neq \cdots \neq \mathbf{k}_{M}$, and

$$
s_{\mathbf{k}_{m}}=\frac{z_{\mathbf{k}_{m}}}{2 \varepsilon_{\mathbf{k}_{m}}-E_{m}},
$$

is a solution of Eq. (136). Interestingly, the function $\Psi^{M}\left(\left\{s_{\mathbf{k}}\right\}\right)$ represents an elementary symmetric function of order $M$. It turns out that these functions are the equivalent of the 
Richardson's solutions in the continuum, i.e.,

$$
H \Psi^{M}\left(\left\{s_{\mathbf{k}}\right\}\right)=\left(\bar{E}+\sum_{m=1}^{M} E_{m}\right) \Psi^{M}\left(\left\{s_{\mathbf{k}}\right\}\right),
$$

with the complex numbers $E_{m}$ satisfying Richardson's equations

$$
1+g \sum_{\mathbf{k}} \frac{1}{2 \varepsilon_{\mathbf{k}}-E_{m}}+2 g \sum_{\ell(\neq m)=1}^{M} \frac{1}{E_{m}-E_{\ell}}=0 .
$$

\section{Conclusions}

The benefits of having exact solutions to problems involving strongly interacting manyparticle systems are difficult to overstate. New exactly(or quasiexactly)-solvable models are always a unique tool to better understand physical phenomena characterized by nonlinear and nonperturbative effects. Moreover, exactly-solvable models are excellent testing grounds for approximations to the many-body problem. In the present work we have explored a generalized Gaudin algebra, whose invariants provide the generating function for integrable quantum Hamiltonians called $X Y Z$ Gaudin models. These quantum invariants can be simultaneously diagonalized using the Bethe ansatz. Different representations of the generators of the generalized Gaudin algebra realize many well-known physical Hamiltonians including the Bardeen-Cooper-Schrieffer, Suhl-Matthias-Walker, interacting boson model of nuclei, Lipkin-Meshkov-Glick, several BEC models, generalized Dicke, spinboson, a new Kondo-like, and many other models not yet exploited in the literature. In this way, we have identified the underlying algebraic structure, thus providing a unifying framework. An advantage of the Bethe ansatz for the Gaudin models is that the physical interpretation of their eigenfunctions is straightforward. The built-in correlation physics is so transparent that they could have well been chosen as exact variational states.

An important question concerns the differences between mean-field approximations to the eigenfunctions of the Gaudin models and their exact solutions in the thermodynamic limit. Here, we briefly discussed issues related to the quantum critical behavior of these models. In particular, we analyzed the nature of the transition between the superconducting and Fermi liquid phases in the BCS model. We concluded that it is of the KosterlitzThouless type independently of the space dimensionality of the lattice.

A number of applications have been presented with the intention of illustrating the variety of physics problems described by microscopic Hamiltonians which belong to the class of $X X Z$ Gaudin models. We have shown that the Lipkin-Meshkov-Glick model, widely used in nuclear physics and, more recently, in connection with quantum information theory, is exactly-solvable. Our proof seems to complete the work initiated in Ref. [46] to the whole parameter space (including the sector $W^{2}<V^{2}$ ). The exact solvability of the two-level boson Hamiltonians given in 6.3 comprises three important models, the LMG, the IBM (describing the transition from the $U(5)$ to the $O(6)$ dynamical symmetries), and the two Josephson-coupled BECs. All of them are, therefore, characterized by the same physics. 
We have also shown that the $X Y Z$ Gaudin equation, Eq. (6), results from the use of the Jacobi identities for the generators of the algebra, i.e., it is a property of the algebra. For the $X X Z$ case, we have derived a family of antisymmetric solutions which includes the rational, trigonometric, hyperbolic, and Richardson's solution as special cases. Indeed, we have proved that the latter is a reparametrization of any of the other three.

Solving efficiently the nonlinear Bethe equations, which provide the necessary spectral parameters, is an important technical issue. In this regard, knowing the strong- and weakcoupling limits of those equations help to reduce the complexity of the task. In the stronginteracting limit this analysis was previously done in Ref. [55]. In Appendix A we have shown that the weakly-coupled limit solutions of the Bethe equations are given by the roots of Laguerre polynomials, by transforming those equations into a generalized Stieltjes equation. In this way, we proved that the Bethe ansatz encompasses all possible eigenstates, and does not provide spurious solutions for finite couplings.

In this paper we concentrated on the spectrum and eigenfunctions of generalized Gaudin models. Certain static correlators for the Gaudin magnet and the Richardson model were studied in Refs. [56-58]. Computation of correlation functions for the generalized $X Y Z$ Gaudin models, either static or dynamic, constitute a more demanding task and is a subject of future research.

\section{Acknowledgements}

This work was supported by the US DOE through Contract No. W-7405-ENG-36, by the Spanish DGI under grant BFM2003-05316-C02-02 and by the Fund for Scientific Research, Flanders (Belgium). Fruitful discussions with C.D. Batista, V.G. Guerguiev, I. Martin, S. Trugman and D. Van Neck are acknowledged.

\section{Appendix A. The weakly interacting limit}

The Bethe equations, Eq. (41), can be expressed in terms of the parametrization of Eq. (14) as

$$
\begin{aligned}
& \frac{1-2 g s\left(\sum_{\mathbf{j} \in \mathcal{T}} d_{\mathbf{j}}+M-1\right)}{1+s t_{\ell}^{2}}+2 g \sum_{\mathbf{j} \in \mathcal{T}} \frac{d_{\mathbf{j}}}{t_{\ell}-t_{\mathbf{j}}}+2 g \sum_{n(\neq \ell)=1}^{M} \frac{1}{t_{\ell}-t_{n}}=0, \\
& \quad \ell=1, \ldots, M .
\end{aligned}
$$

In the limit $g \rightarrow 0$ one recovers the noninteracting model, with the variables $t_{\ell}$ converging to the parameter values $t_{\mathbf{j}}$, depending on the corresponding distribution of the particles or spins. Therefore one can make the substitution

$$
t_{\ell}=t_{\mathbf{j}_{\ell}}+g x_{\ell}
$$


where $\mathbf{j}_{\ell}$ is the index of the parameter value to which $t_{\ell}$ converges. Then Eq. (A.1) can be rewritten up to first order in $g$ as

$$
\frac{2 d_{\mathbf{j} \ell}}{x_{\ell}}+\sum_{n, \mathbf{j}_{n}=\mathbf{j}_{\ell}, n \neq \ell} \frac{2}{x_{\ell}-x_{n}}=-\frac{1+2 g \alpha_{\mathbf{j} \ell}}{1+s t_{\mathbf{j}_{\ell}}^{2}}
$$

with $\alpha_{\mathbf{j} \ell}$ independent of the variables $x_{\ell}$ :

$$
\alpha_{\mathbf{j}_{\ell}}=\sum_{\mathbf{j} \in \mathcal{T}, \mathbf{j} \neq \mathbf{j}_{\ell}} d_{\mathbf{j}} \frac{1+s t_{\mathbf{j} \ell} t_{\mathbf{j}}}{t_{\mathbf{j} \ell}-t_{\mathbf{j}}}+\sum_{n=1, \mathbf{j}_{n} \neq \mathbf{j}_{\ell}}^{M} \frac{1+s t_{\mathbf{j}_{\ell}} t_{\mathbf{j}_{n}}}{t_{\mathbf{j} \ell}-t_{\mathbf{j}_{n}}}-s\left(d_{\mathbf{j} \ell}+N_{\mathbf{j}_{\ell}}-1\right),
$$

and where $N_{\mathbf{j} \ell}$ is the number of variables $t_{n}$ that cluster around the parameter $t_{\mathbf{j} \ell}$.

Eq. (A.3) can be transformed into a Generalized Stieltjes equation [59], with the solutions given by:

$$
x_{\ell}=-\frac{1+s t_{\mathbf{j}_{\ell}}^{2}}{1+2 g \alpha_{\mathbf{j} \ell}} r_{l}
$$

where the $r_{l}$ are the roots of the associated Laguerre polynomials $L_{N}^{k}$, with $k=2 d_{\mathbf{j} \ell}-1$ and $N=N_{\mathbf{j}_{\ell}}$. Note that the resulting values for the variables $t_{\ell}$ are correct up to second order in $g$ :

$$
t_{\ell}=t_{\mathbf{j} \ell}+g \frac{\mathrm{f}\left(t_{\mathbf{j}_{\ell}}\right)}{1+2 g \alpha_{\mathbf{j}_{\ell}}} r_{l} .
$$

The variables $r_{l}$ will be real for $d_{\mathbf{j} \ell}>0$ (with $\operatorname{su}(1,1)$ realizations, typical for bosons), while for $d_{\mathbf{j}_{\ell}}<0$ (with $s u(2)$ realizations, typical for fermions) the variables $x_{\ell}$ will come in complex conjugated pairs, except for one real value in case of an odd number of variables per cluster.

Because a polynomial of order $N$ has a unique set of $N$ roots, the weakly interacting limit establishes a one-to-one mapping between the noninteracting solutions (defined by the number of variables clustered around each parameter $t_{\mathbf{j}}$ ) and the solutions at finite values of $g$.

\section{References}

[1] T. Senthil, et al., Science 303 (2004) 1490.

[2] M. Gaudin, La Fonction d'Onde de Bethe, Masson, Paris, 1983.

[3] A.G. Ushveridze, Quasi-Exactly Solvable Models in Quantum Mechanics, IOP, Bath, 1994.

[4] C.D. Batista, G. Ortiz, Phys. Rev. Lett. 85 (2000) 4755.

[5] J. Bardeen, L.N. Cooper, J.R. Schrieffer, Phys. Rev. 108 (1957) 1175.

[6] H. Suhl, B.T. Matthias, L.R. Walker, Phys. Rev. Lett. 3 (1959) 552.

[7] H. Lipkin, N. Meshkov, A. Glick, Nucl. Phys. 62 (1965) 188.

[8] R.H. Dicke, Phys. Rev. 93 (1954) 99.

[9] J. Dukelsky, C. Esebbag, P. Schuck, Phys. Rev. Lett. 87 (2001) 066403.

[10] L. Amico, A. Di Lorenzo, A. Osterloh, Phys. Rev. Lett. 86 (2001) 5759.

[11] R.W. Richardson, cond-mat/0203512.

[12] C.D. Batista, G. Ortiz, Adv. Phys. 53 (2004) 1, cond-mat/0207106.

[13] F. Iachello, A. Arima, The Interacting Boson Model, Cambridge Univ. Press, Cambridge, 1987. 
[14] S. Kohler, F. Sols, Phys. Rev. Lett. 89 (2002) 60403.

[15] R. Somma, G. Ortiz, H. Barnum, E. Knill, L. Viola, Phys. Rev. A 70 (2004) 042311.

[16] R. Gilmore, Catastrophe Theory for Scientists and Engineers, Wiley-Interscience, New York, 1981.

[17] J.M. Kosterlitz, D.J. Thouless, J. Phys. C 6 (1973) 1181.

[18] Another generalization of the $X X Z$ model can be found in A. Di Lorenzo, et al., Nucl. Phys. B 644 (2002) 409.

[19] M. Gaudin, J. Physique 37 (1976) 1087.

[20] E.K. Sklyanin, T. Takebe, Commun. Math. Phys. 204 (1999) 1.

[21] M.D. Gould, Y.-Z. Zhang, S.-Y. Zhao, Nucl. Phys. B 630 (2002) 492.

[22] J.M. Román, G. Sierra, J. Dukelsky, Phys. Rev. B 67 (2003) 064510.

[23] H. Kamerlingh Onnes, Comm. Leiden 119b, 120b, 122b, 124c (1911); J. de Noble, Phys. Today 49 (9) (1996) 40.

[24] See, for example, M. Tinkham, Introduction to Superconductivity, McGraw-Hill, New York, 1996.

[25] A. Bohr, B.R. Mottelson, D. Pines, Phys. Rev. 110 (1958) 936.

[26] R.W. Richardson, Phys. Lett. 3 (1963) 277;

R.W. Richardson, Phys. Lett. 5 (1963) 82;

R.W. Richardson, Phys. Lett. 14 (1965) 325;

R.W. Richardson, J. Math. Phys. 6 (1965) 1034;

R.W. Richardson, Phys. Rev. 144 (1966) 874;

R.W. Richardson, Phys. Rev. 159 (1967) 792;

R.W. Richardson, N. Sherman, Nucl. Phys. 52 (1964) 221.

[27] M.C. Cambiaggio, A.M.F. Rivas, M. Saraceno, Nucl. Phys. A 424 (1997) 157.

[28] J. von Delft, D.C. Ralph, Phys. Rep. 345 (2001) 61.

[29] D. Gobert, M. Schechter, U. Schollwock, J. von Delft, cond-mat/0404614.

[30] R.W. Richardson, Phys. Rev. 141 (1966) 949.

[31] G. Sierra, J. Dukelsky, G.G. Dussel, J. von Delft, F. Braun, Phys. Rev. B 61 (2000) R11890.

[32] S. Rombouts, D. Van Neck, J. Dukelsky, Phys. Rev. C 69 (2004) 061303(R) .

[33] R.W. Richardson, J. Math. Phys. 18 (1977) 1802.

[34] J.M. Román, G. Sierra, J. Dukelsky, Nucl. Phys. B 634 (2002) 483.

[35] H.R. Krishnamurthy, B.S. Shastry, Phys. Rev. Lett. 84 (2000) 4918.

[36] J. Dukelsky, C. Esebbag, S. Pittel, Phys. Rev. Lett. 88 (2002) 062501.

[37] L. Amico, A. Di Lorenzo, A. Osterloh, Nucl. Phys. B 614 (2001) 449.

[38] F. Pan, J.P. Draayer, W.E. Ormand, Phys. Lett. B 422 (1998) 1.

[39] S. Rombouts, D. Van Neck, K. Peirs, L. Pollet, Mod. Phys. Lett. A 17 (2002) 1899.

[40] D. Van Neck, Y. Dewulf, M. Waroquier, Phys. Rev. A 63 (2001) 062107.

[41] C.K. Law, H. Pu, N.P. Bigelow, Phys. Rev. Lett. 81 (1998) 5257.

[42] R.W. Richardson, J. Math. Phys. 9 (1968) 1327.

[43] J. Dukelsky, P. Schuck, Phys. Rev. Lett. 86 (2001) 4207.

[44] J. Dukelsky, S. Pittel, Phys. Rev. Lett. 86 (2001) 4791.

[45] J.M. Arias, J. Dukelsky, J.E. García-Ramos, Phys. Rev. Lett. 91 (2003) 162502.

[46] F. Pan, J.P. Draayer, Phys. Lett. B 451 (1999) 1.

[47] B.D. Josephson, Phys. Lett. 1 (1962) 251.

[48] H.-Q. Zhou, J. Links, R.H. McKenzie, X.-W. Guan, J. Phys A 36 (2003) L113.

[49] G. Falci, R. Fazio, A. Mastellone, Phys. Rev. B 67 (2003) 132501.

[50] B. Jurčo, J. Math. Phys. 30 (1989) 1739.

[51] A. Kundu, J. Phys. A 37 (2004) L281.

[52] J. Dukelsky, G.G. Dussel, C. Esebbag, S. Pittel, Phys. Rev. Lett. 93 (2004) 050403.

[53] J. Kondo, Prog. Theor. Phys. 32 (1964) 37.

[54] N. Andrei, Phys. Rev. Lett. 45 (1980) 379;

P.B. Wiegmann, Sov. Phys. JETP Lett. 31 (1980) 392.

[55] E.A. Yuzbashyan, A.A. Baytin, B.L. Altshuler, Phys. Rev. B 68 (2003) 214509.

[56] E.K. Sklyanin, Lett. Math. Phys. 47 (1999) 275.

[57] L. Amico, A. Osterloh, Phys. Rev. Lett. 88 (2002) 127003.

[58] H.-Q. Zhou, J. Links, R.H. McKenzie, M.D. Gould, Phys. Rev. B 65 (2002) 060502.

[59] B.S. Shastry, A. Dhar, J. Phys. A: Math. Gen. 34 (2001) 6197. 